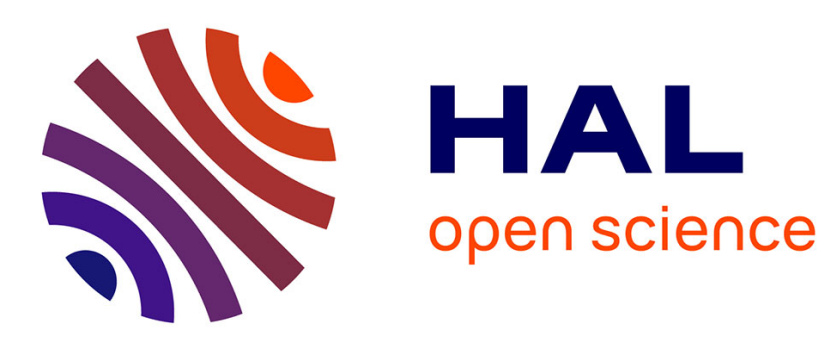

\title{
Permanent regimes for the Vlasov-Maxwell equations with specular boundary conditions
}

\author{
Mihai Bostan
}

\section{To cite this version:}

Mihai Bostan. Permanent regimes for the Vlasov-Maxwell equations with specular boundary conditions. 2009. hal-00357218

\section{HAL Id: hal-00357218 \\ https://hal.science/hal-00357218}

Preprint submitted on 30 Jan 2009

HAL is a multi-disciplinary open access archive for the deposit and dissemination of scientific research documents, whether they are published or not. The documents may come from teaching and research institutions in France or abroad, or from public or private research centers.
L'archive ouverte pluridisciplinaire HAL, est destinée au dépôt et à la diffusion de documents scientifiques de niveau recherche, publiés ou non, émanant des établissements d'enseignement et de recherche français ou étrangers, des laboratoires publics ou privés. 


\title{
Permanent regimes for the Vlasov-Maxwell equations with specular boundary conditions
}

\author{
Mihai Bostan *
}

(January 26, 2009)

\begin{abstract}
The subject matter of this paper concerns the existence of permanent regimes (i.e., stationary or time periodic solutions) for the Vlasov-Maxwell system in a bounded domain. We are looking for equilibrium configurations by imposing specular boundary conditions. The main difficulty is the treatment of such boundary conditions. Our analysis relies on perturbative techniques, based on uniform a priori estimates.
\end{abstract}

Keywords: Vlasov-Maxwell equations, Specular boundary conditions, Permanent regimes.

AMS classification: 35F30, 35L40.

\section{Introduction}

In this paper we construct weak solutions for the Vlasov-Maxwell equations in a bounded domain. Our main interest focus on permanent regimes: stationary or time periodic solutions satisfying specular boundary conditions.

*Laboratoire de Mathématiques de Besançon, UMR CNRS 6623, Université de Franche-Comté, 16 route de Gray, 25030 Besançon Cedex France. E-mail: mbostan@univ-fcomte.fr 
The Vlasov equation describes the dynamics of a population of charged particles, in terms of a particle density $f=f(t, x, p) \geq 0$ depending on time $t \in \mathbb{R}$, position $x \in \Omega$ and momentum $p \in \mathbb{R}^{3}$. Here $\Omega$ is a bounded open subset of $\mathbb{R}^{3}$ with regular boundary $\partial \Omega$. We introduce the standard notations

$$
\Sigma=\partial \Omega \times \mathbb{R}^{3}, \quad \Sigma^{ \pm}=\{(x, p) \in \Sigma: \pm(v(p) \cdot n(x))>0\}
$$

where $n(x)$ is the unit outward normal to $\partial \Omega$ at $x$ and $v(p)$ is the velocity function associated to the kinetic energy function $\mathcal{E}(p)$ by $v(p)=\nabla_{p} \mathcal{E}(p), p \in \mathbb{R}^{3}$. These functions are given in the classical case by

$$
\mathcal{E}(p)=\frac{|p|^{2}}{2 m}, \quad v(p)=\frac{p}{m}
$$

and in the relativistic case by

$$
\mathcal{E}(p)=m c^{2}\left(\left(1+\frac{|p|^{2}}{m^{2} c^{2}}\right)^{1 / 2}-1\right), v(p)=\frac{p}{m}\left(1+\frac{|p|^{2}}{m^{2} c^{2}}\right)^{-1 / 2}
$$

where $m$ is the particle mass, $c$ is the light speed in the vacuum. The motion of the particle population, with charge $q$ and number density $f$, under the action of the electro-magnetic field $(E(t, x), B(t, x))$ is given by the Vlasov equation

$$
\partial_{t} f+v(p) \cdot \nabla_{x} f+q(E(t, x)+v(p) \wedge B(t, x)) \cdot \nabla_{p} f=0, \quad(t, x, p) \in \mathbb{R} \times \Omega \times \mathbb{R}^{3}
$$

We are looking for densities $f$ satisfying specular boundary conditions

$$
f(t, x, p)=f(t, x, R(x) p), \quad(t, x, p) \in \mathbb{R} \times \Sigma^{-}
$$

where $R(x)=I-2 n(x) \otimes n(x)$ is the symmetry with respect to the plane orthogonal to $n(x)$

$$
R(x) p=p-2(p \cdot n(x)) n(x), \quad(x, p) \in \Sigma .
$$

The evolution of the self-consistent electro-magnetic field is given by the Maxwell equations

$$
\begin{aligned}
\partial_{t} E-c^{2} \operatorname{curl}_{x} B & =-\frac{j}{\varepsilon_{0}}, \quad \partial_{t} B+\operatorname{curl}_{x} E=0, \quad(t, x) \in \mathbb{R} \times \Omega \\
\operatorname{div}_{x} E & =\frac{\rho}{\varepsilon_{0}}, \quad \operatorname{div}_{x} B=0, \quad(t, x) \in \mathbb{R} \times \Omega
\end{aligned}
$$

with the Silver-Müler boundary condition

$$
n(x) \wedge E(t, x)+c n(x) \wedge(n(x) \wedge B(t, x))=h(x), \quad(t, x) \in \mathbb{R} \times \partial \Omega
$$


where $\varepsilon_{0}$ is the permittivity of the vacuum, $\rho(t, x)=q \int_{\mathbb{R}^{3}} f(t, x, p) \mathrm{d} p$ is the charge density, $j(t, x)=q \int_{\mathbb{R}^{3}} v(p) f(t, x, p) \mathrm{d} p$ is the current density and $h$ is a given tangential field on the boundary $\partial \Omega$ i.e., $(n(x) \cdot h(x))=0, x \in \partial \Omega$, such that $H=$ $\int_{\partial \Omega}|h(x)|^{2} \mathrm{~d} \sigma<+\infty$. The system (3), (4), (5), (6), (7) is called the Vlasov-Maxwell problem. By weak solution we understand solution in the distribution sense.

Definition 1.1 Assume that $h \in L^{2}(\partial \Omega)^{3},\left.(n \cdot h)\right|_{\partial \Omega}=0$. We say that $(f, E, B) \in$ $L_{\text {loc }}^{1}\left(\mathbb{R} ; L^{1}\left(\Omega \times \mathbb{R}^{3}\right)\right) \times L_{\text {loc }}^{1}\left(\mathbb{R} ; L^{2}(\Omega)^{3}\right)^{2}$ is a $T$ periodic weak solution for the VlasovMaxwell problem iff

$$
\int_{0}^{T} \int_{\Omega} \int_{\mathbb{R}^{3}} f(t, x, p)\left(\partial_{t} \theta+v(p) \cdot \nabla_{x} \theta+q(E(t, x)+v(p) \wedge B(t, x)) \cdot \nabla_{p} \theta\right) \mathrm{d} p \mathrm{~d} x \mathrm{~d} t=0
$$

for any $T$ periodic function $\theta \in C^{1}\left(\mathbb{R} \times \bar{\Omega} \times \mathbb{R}^{3}\right)$ satisfying $\theta(t, x, p)=\theta(t, x, R(x) p)$, $(t, x, p) \in \mathbb{R} \times \Sigma^{+}$and

$$
\begin{aligned}
& \int_{0}^{T} \int_{\Omega}\left\{E(t, x) \cdot \partial_{t} \varphi+c^{2} B(t, x) \cdot \partial_{t} \psi+c^{2}\left(B(t, x) \cdot \operatorname{curl}_{x} \varphi-E(t, x) \cdot \operatorname{curl}_{x} \psi\right)\right\} \mathrm{d} x \mathrm{~d} t \\
+ & c \int_{0}^{T} \int_{\partial \Omega}(n(x) \wedge \varphi) \cdot h(x) \mathrm{d} \sigma \mathrm{d} t-\frac{1}{\varepsilon_{0}} \int_{0}^{T} \int_{\Omega} \int_{\mathbb{R}^{3}}(v(p) \cdot \varphi) f(t, x, p) \mathrm{d} p \mathrm{~d} x \mathrm{~d} t=0
\end{aligned}
$$

for all $T$ periodic fields $\varphi, \psi \in C^{1}(\mathbb{R} \times \bar{\Omega})^{3}$ satisfying $n(x) \wedge \varphi(t, x)-c n(x) \wedge(n(x) \wedge$ $\psi(t, x))=0,(t, x) \in \mathbb{R} \times \partial \Omega$.

Remark 1.1 In order to also satisfy the divergence constraint (6) it is convenient to solve the perturbed periodic problem (which is obtained by replacing all the time derivatives $\partial_{t}$ by $\alpha+\partial_{t}$ )

$$
\left\{\begin{array}{l}
\alpha f(t, x, p)+\partial_{t} f+v(p) \cdot \nabla_{x} f+q(E(t, x)+v(p) \wedge B(t, x)) \cdot \nabla_{p} f=0 \\
\alpha E(t, x)+\partial_{t} E-c^{2} \operatorname{curl}_{x} B=-\frac{j(t, x)}{\varepsilon_{0}} \\
\alpha B(t, x)+\partial_{t} B+\operatorname{curl}_{x} E=0
\end{array}\right.
$$

for any $\alpha>0$ and then to pass to the limit when $\alpha$ goes to 0 . Indeed, in this case it is easily seen that

$$
\alpha \operatorname{div}_{x} B+\partial_{t} \operatorname{div}_{x} B=0
$$

and therefore, by time periodicity one gets $\operatorname{div}_{x} B=0$. Similarly, using the continuity equation

$$
\alpha \rho(t, x)+\partial_{t} \rho+\operatorname{div}_{x} j=0
$$


we deduce that

$$
\alpha\left(\operatorname{div}_{x} E-\frac{\rho}{\varepsilon_{0}}\right)+\partial_{t}\left(\operatorname{div}_{x} E-\frac{\rho}{\varepsilon_{0}}\right)=0
$$

which implies by time periodicity that $\operatorname{div}_{x} E=\frac{\rho}{\varepsilon_{0}}$. For simplifying our computations we skip these details: we perform the computations with $\alpha=0$, assuming that the divergence constraints hold true (the reader can convince himself that similar results hold when keeping $\alpha>0$ in the equations).

The existence of global weak solution for the Vlasov-Maxwell system in three dimensions was obtained by DiPerna and Lions [9]. The global existence of strong solutions is still an open problem. Results for the relativistic case were obtained by Glassey and Schaeffer [11], [12], Glassey and Strauss [13], [14], Klainerman and Staffilani [18], Bouchut, Golse and Pallard [7].

Neglecting the relativistic corrections and the magnetic field leads to the VlasovPoisson problem. This model is justified by studying the asymptotic behaviour of the relativistic Vlasov-Maxwell problem when the particle velocities are small with respect to the light speed [8], [24], [19], [5].

The Cauchy problem for the free space Vlasov-Poisson system is now well understood, see Arseneev [1], Horst and Hunze [17] for weak solutions, Ukai and Okabe [26], Pfaffelmoser [22], Bardos and Degond [2], Schaeffer [25], Lions and Perthame [20] for strong solutions.

For applications (vacuum diodes, tube discharges, satellite ionization, thrusters, etc.) the boundary conditions need to be considered [3], [16]. The stationary problems were studied by Greengard and Raviart [15], Poupaud [23]. Results for the time periodic case can be found in [4], [6].

Our main result is the following

Theorem 1.1 Assume that $\Omega$ is a bounded open set of $\mathbb{R}^{3}$ with smooth boundary, strictly star-shaped. Let $g=g(t, x, p)$ be a $T$ periodic non negative bounded function on $\mathbb{R} \times \Sigma^{-}$and $h=h(x)$ be a tangential field on $\partial \Omega$ verifying

$$
\begin{gathered}
M^{-}=\int_{0}^{T} \int_{\Sigma^{-}}|(v(p) \cdot n(x))| g(t, x, p) \mathrm{d} p \mathrm{~d} \sigma \mathrm{d} t<+\infty \\
K^{-}=\int_{0}^{T} \int_{\Sigma^{-}}|(v(p) \cdot n(x))| \mathcal{E}(p) g(t, x, p) \mathrm{d} p \mathrm{~d} \sigma \mathrm{d} t<+\infty
\end{gathered}
$$




$$
H=\int_{\partial \Omega}|h(x)|^{2} \mathrm{~d} \sigma<+\infty .
$$

Then there is a weak $T$ periodic solution $(f, E, B)$ for the Vlasov-Maxwell problem (3), (4), (5), (6), (7), with traces $\gamma^{ \pm} f$ on $\mathbb{R} \times \Sigma^{ \pm}$, tangential traces $(n \wedge E, n \wedge B)$ and normal traces $(n \cdot E, n \cdot B)$ on $\mathbb{R} \times \partial \Omega$ satisfying

$$
\begin{gathered}
\int_{0}^{T} \int_{\Sigma^{ \pm}}|(v(p) \cdot n(x))| \gamma^{ \pm} f \mathrm{~d} p \mathrm{~d} \sigma \mathrm{d} t=\int_{0}^{T} \int_{\Sigma^{-}}|(v(p) \cdot n(x))| g \mathrm{~d} p \mathrm{~d} \sigma \mathrm{d} t=M^{-} \\
\int_{0}^{T} \int_{\Omega} \int_{\mathbb{R}^{3}} \mathcal{E}(p) f(t, x, p) \mathrm{d} p \mathrm{~d} x \mathrm{~d} t+\frac{\varepsilon_{0}}{2} \int_{0}^{T} \int_{\Omega}\left(|E|^{2}+c^{2}|B|^{2}\right) \mathrm{d} x \mathrm{~d} t \leq C_{1} \\
\int_{0}^{T} \int_{\Sigma}|(v(p) \cdot n(x))| \mathcal{E}(p) \gamma f(t, x, p) \mathrm{d} p \mathrm{~d} \sigma \mathrm{d} t \leq C_{1} \\
\frac{\varepsilon_{0}}{2} \int_{0}^{T} \int_{\partial \Omega}\left(|n \wedge E|^{2}+c^{2}|n \wedge B|^{2}\right) \mathrm{d} \sigma \mathrm{d} t+\frac{\varepsilon_{0}}{2} \int_{0}^{T} \int_{\partial \Omega}(n \cdot E)^{2}+c^{2}(n \cdot B)^{2} \mathrm{~d} \sigma \mathrm{d} t \leq C_{1}
\end{gathered}
$$

for some constant $C_{1}$ depending on $\Omega, T, H,\|g\|_{L^{\infty}\left(\mathbb{R} \times \Sigma^{-}\right)}, M^{-}, K^{-}$.

We also study the Vlasov-Maxwell problem with perfect conducting boundary conditions

$$
n \wedge E=0, \quad n \cdot B=0, \quad(t, x) \in \mathbb{R} \times \partial \Omega .
$$

The second perfect conducting boundary condition in (11) is a consequence of the first perfect conducting boundary condition in (11) and the time periodicity. Indeed, as before, replacing $\partial_{t}$ by $\alpha+\partial_{t}$ leads to

$$
\alpha B+\partial_{t} B+\operatorname{curl}_{x} E=0, \quad(t, x) \in \mathbb{R} \times \Omega .
$$

Multiplying by $\nabla_{x} \varphi$, where $\varphi \in C^{1}(\bar{\Omega})$ and taking into account that $\operatorname{div}_{x} B=0, n \wedge E=$ 0 yield after integration by parts

$$
\alpha \int_{\partial \Omega}(n(x) \cdot B(t, x)) \varphi \mathrm{d} \sigma+\frac{d}{d t} \int_{\partial \Omega}(n(x) \cdot B(t, x)) \varphi \mathrm{d} \sigma=0 .
$$

Therefore, since $t \rightarrow \int_{\partial \Omega}(n(x) \cdot B(t, x)) \varphi \mathrm{d} \sigma$ is $T$ periodic, one gets $\int_{\partial \Omega}(n(x) \cdot B(t, x)) \varphi(x) \mathrm{d} \sigma=$ $0, t \in \mathbb{R}$, for any $\varphi \in C^{1}(\bar{\Omega})$, saying that $n \cdot B=0$ on $\mathbb{R} \times \partial \Omega$. As said before, we perform our computations only for $\alpha=0$, assuming that the perfect conducting boundary condition $n \cdot B=0$ holds true. We establish the existence result 
Theorem 1.2 Assume that $\Omega$ is a bounded open set of $\mathbb{R}^{3}$ with smooth boundary, strictly star-shaped. Let $g=g(t, x, p)$ be a $T$ periodic non negative bounded function on $\mathbb{R} \times \Sigma^{-}$verifying

$$
\begin{gathered}
M^{-}=\int_{0}^{T} \int_{\Sigma^{-}}|(v(p) \cdot n(x))| g(t, x, p) \mathrm{d} p \mathrm{~d} \sigma \mathrm{d} t<+\infty \\
K^{-}=\int_{0}^{T} \int_{\Sigma^{-}}|(v(p) \cdot n(x))| \mathcal{E}(p) g(t, x, p) \mathrm{d} p \mathrm{~d} \sigma \mathrm{d} t<+\infty .
\end{gathered}
$$

Then there is a weak $T$ periodic solution $(f, E, B)$ for the Vlasov-Maxwell problem (3), (4), (5), (6), (11) satisfying

$$
\begin{gathered}
\int_{0}^{T} \int_{\Sigma^{ \pm}}|(v(p) \cdot n(x))| \gamma^{ \pm} f \mathrm{~d} p \mathrm{~d} \sigma \mathrm{d} t=\int_{0}^{T} \int_{\Sigma^{-}}|(v(p) \cdot n(x))| g \mathrm{~d} p \mathrm{~d} \sigma \mathrm{d} t=M^{-} \\
\int_{0}^{T} \int_{\Omega} \int_{\mathbb{R}^{3}} \mathcal{E}(p) f(t, x, p) \mathrm{d} p \mathrm{~d} x \mathrm{~d} t+\frac{\varepsilon_{0}}{2} \int_{0}^{T} \int_{\Omega}\left(|E|^{2}+c^{2}|B|^{2}\right) \mathrm{d} x \mathrm{~d} t \leq C_{2} \\
\int_{0}^{T} \int_{\Sigma}|(v(p) \cdot n(x))| \mathcal{E}(p) \gamma f(t, x, p) \mathrm{d} p \mathrm{~d} \sigma \mathrm{d} t \leq C_{2} \\
\frac{\varepsilon_{0}}{2} \int_{0}^{T} \int_{\partial \Omega}\left(c^{2}|n \wedge B|^{2}+(n \cdot E)^{2}\right) \mathrm{d} \sigma \mathrm{d} t \leq C_{2}
\end{gathered}
$$

for some constant $C_{2}$ depending on $\Omega, T,\|g\|_{L^{\infty}\left(\mathbb{R} \times \Sigma^{-}\right)}, M^{-}, K^{-}$.

Our paper is organized as follows. We start by constructing $T$ periodic solutions $\left(f_{\varepsilon}, E_{\varepsilon}, B_{\varepsilon}\right)$ for the Vlasov-Maxwell system with the boundary condition (4) replaced by

$$
f(t, x, p)=\varepsilon g(t, x, p)+(1-\varepsilon) f(t, x, R(x) p), \quad(t, x, p) \in \mathbb{R} \times \Sigma^{-}
$$

where $\varepsilon \in] 0,1]$ is a small parameter and $g$ is a $T$ periodic bounded non negative function on $\mathbb{R} \times \Sigma^{-}$such that $M^{-}(g)<+\infty, K^{-}(g)<+\infty$. The existence of such solutions has been established in [6], pp. 660. The key point here is that the boundary condition (12) is of the form

$$
f(t, x, p)=\tilde{g}(t, x, p)+a f(t, x, R(x) p), \quad(t, x, p) \in \mathbb{R} \times \Sigma^{-}
$$

where $0 \leq a<1$. Section 2 is devoted to uniform estimates with respect to the small parameter $\varepsilon$. In the last section we appeal to compactness arguments, in order to construct $T$ periodic weak solutions for the Vlasov-Maxwell system with specular boundary condition for particles and Silver-Müler or perfect conducting boundary condition for the electro-magnetic field. 


\section{A priori estimates}

In this section we establish uniform estimates with respect to $\varepsilon \in] 0,1]$ for the periodic solutions $\left(f_{\varepsilon}, E_{\varepsilon}, B_{\varepsilon}\right)_{\varepsilon>0}$ of $(3),(12),(5),(6),(7)$. We perform these computations only for smooth solutions, compactly supported in momentum. The general case follows by standard arguments using regularization and weak stability, see [6]. We skip these details. We appeal to the conservation of the mass, momentum and total energy. We need the following easy lemma.

Lemma 2.1 Let $f=f(x, p), g=g(x, p),(x, p) \in \Sigma$ be non negative functions satisfying

$$
f(x, p)=\varepsilon g(x, p)+(1-\varepsilon) f(x, R(x) p), \quad(x, p) \in \Sigma^{-} .
$$

We assume that $F=F(x,|p|)$ is a non negative function such that

$$
\begin{gathered}
\int_{\partial \Omega} \int_{\mathbb{R}^{3}}|(v(p) \cdot n(x))| F(x,|p|) f(x, p) \mathrm{d} p \mathrm{~d} \sigma<+\infty \\
\int_{\partial \Omega} \int_{\mathbb{R}^{3}}(v(p) \cdot n(x))_{-} F(x,|p|) g(x, p) \mathrm{d} p \mathrm{~d} \sigma<+\infty .
\end{gathered}
$$

Then for a.a. $x \in \partial \Omega$ we have

$$
\begin{aligned}
\int_{\mathbb{R}^{3}}(v(p) \cdot n(x)) F(x,|p|) f(x, p) \mathrm{d} p & =\varepsilon \int_{\mathbb{R}^{3}}(v(p) \cdot n(x))_{+} F(x,|p|) f(x, p) \mathrm{d} p \\
& -\varepsilon \int_{\mathbb{R}^{3}}(v(p) \cdot n(x))_{-} F(x,|p|) g(x, p) \mathrm{d} p
\end{aligned}
$$

where $(\cdot)_{ \pm}$stands for the positive/negative part.

Proof. Pick any function $\varphi \in C(\partial \Omega)$ and observe that

$$
\begin{aligned}
\int_{\partial \Omega} \int_{\mathbb{R}^{3}}(v \cdot n) \varphi F f \mathrm{~d} p \mathrm{~d} \sigma & =\int_{\partial \Omega} \int_{\mathbb{R}^{3}}(v(p) \cdot n(x))_{+} \varphi(x) F(x,|p|) f \mathrm{~d} p \mathrm{~d} \sigma \\
& -\int_{\partial \Omega} \int_{\mathbb{R}^{3}}(v(p) \cdot n(x))_{-} \varphi(x) F(x,|p|) f \mathrm{~d} p \mathrm{~d} \sigma \\
& =\int_{\partial \Omega} \int_{\mathbb{R}^{3}}(v(p) \cdot n(x))_{+} \varphi(x) F(x,|p|) f \mathrm{~d} p \mathrm{~d} \sigma \\
& -\varepsilon \int_{\partial \Omega} \int_{\mathbb{R}^{3}}(v(p) \cdot n(x))_{-} \varphi(x) F(x,|p|) g \mathrm{~d} p \mathrm{~d} \sigma \\
& -(1-\varepsilon) \int_{\partial \Omega} \int_{\mathbb{R}^{3}}(v(p) \cdot n(x))_{-} \varphi F f(x, R(x) p) \mathrm{d} p \mathrm{~d} \sigma \cdot(13)
\end{aligned}
$$


Notice that we have $|R(x) p|=|p|,(v(p) \cdot n(x))=-(v(R(x) p) \cdot n(x))$ implying that

$$
(v(p) \cdot n(x))_{-}=(v(R(x) p) \cdot n(x))_{+} .
$$

Performing the change of variable $q=R(x) p$ yields the equality

$$
\begin{aligned}
& \int_{\partial \Omega} \int_{\mathbb{R}^{3}}(v(p) \cdot n(x))-\varphi(x) F(x,|p|) f(x, R(x) p) \mathrm{d} p \mathrm{~d} \sigma \\
= & \int_{\partial \Omega} \int_{\mathbb{R}^{3}}(v(q) \cdot n(x))+\varphi(x) F(x,|q|) f(x, q) \mathrm{d} q \mathrm{~d} \sigma .
\end{aligned}
$$

Combining (13), (14) implies

$$
\begin{aligned}
\int_{\partial \Omega} \int_{\mathbb{R}^{3}}(v \cdot n) \varphi F f \mathrm{~d} p \mathrm{~d} \sigma & =\varepsilon \int_{\partial \Omega} \int_{\mathbb{R}^{3}}(v(p) \cdot n(x))_{+} \varphi(x) F(x,|p|) f(x, p) \mathrm{d} p \mathrm{~d} \sigma \\
& -\varepsilon \int_{\partial \Omega} \int_{\mathbb{R}^{3}}(v(p) \cdot n(x))_{-} \varphi(x) F(x,|p|) g(x, p) \mathrm{d} p \mathrm{~d} \sigma
\end{aligned}
$$

and therefore one gets for a.a. $x \in \partial \Omega$

$$
\begin{aligned}
\int_{\mathbb{R}^{3}}(v(p) \cdot n(x)) F(x,|p|) f(x, p) \mathrm{d} p & =\varepsilon \int_{\mathbb{R}^{3}}(v(p) \cdot n(x))_{+} F(x,|p|) f(x, p) \mathrm{d} p \\
& -\varepsilon \int_{\mathbb{R}^{3}}(v(p) \cdot n(x))_{-} F(x,|p|) g(x, p) \mathrm{d} p .
\end{aligned}
$$

The first uniform estimate comes by the mass conservation.

Proposition 2.1 For any $\varepsilon \in] 0,1]$, the $T$ periodic particle density $f_{\varepsilon}$ satisfies

$$
\int_{0}^{T} \int_{\partial \Omega} \int_{\mathbb{R}^{3}}(v(p) \cdot n(x))_{ \pm} f_{\varepsilon}(t, x, p) \mathrm{d} p \mathrm{~d} \sigma \mathrm{d} t=M^{-} .
$$

Proof. Integrating the Vlasov equation (3) on $[0, T] \times \Omega \times \mathbb{R}^{3}$ yields

$$
\int_{0}^{T} \int_{\partial \Omega} \int_{\mathbb{R}^{3}}(v(p) \cdot n(x)) f_{\varepsilon}(t, x, p) \mathrm{d} p \mathrm{~d} \sigma \mathrm{d} t=0 .
$$

Applying Lemma 2.1 with $F=1$ implies

$$
\begin{aligned}
\int_{0}^{T} \int_{\partial \Omega} \int_{\mathbb{R}^{3}}(v(p) \cdot n(x)) f_{\varepsilon}(t, x, p) \mathrm{d} p \mathrm{~d} \sigma \mathrm{d} t & =\varepsilon \int_{0}^{T} \int_{\partial \Omega} \int_{\mathbb{R}^{3}}(v(p) \cdot n(x))_{+} f_{\varepsilon}(t, x, p) \mathrm{d} p \mathrm{~d} \sigma \mathrm{d} t \\
& -\varepsilon \int_{0}^{T} \int_{\partial \Omega} \int_{\mathbb{R}^{3}}(v(p) \cdot n(x))_{-} g(t, x, p) \mathrm{d} p \mathrm{~d} \sigma \mathrm{d} t .
\end{aligned}
$$

We deduce that

$$
\int_{0}^{T} \int_{\partial \Omega} \int_{\mathbb{R}^{3}}(v(p) \cdot n(x))_{+} f_{\varepsilon}(t, x, p) \mathrm{d} p \mathrm{~d} \sigma \mathrm{d} t=\int_{0}^{T} \int_{\partial \Omega} \int_{\mathbb{R}^{3}}(v(p) \cdot n(x))_{-} g(t, x, p) \mathrm{d} p \mathrm{~d} \sigma \mathrm{d} t=M^{-} .
$$


Using the boundary condition (12) we obtain also

$$
\begin{aligned}
\int_{0}^{T} \int_{\partial \Omega} \int_{\mathbb{R}^{3}}(v \cdot n)_{-} f_{\varepsilon} \mathrm{d} p \mathrm{~d} \sigma \mathrm{d} t & =\int_{0}^{T} \int_{\partial \Omega} \int_{\mathbb{R}^{3}}(v \cdot n)_{-}\left(\varepsilon g+(1-\varepsilon) f_{\varepsilon}(t, x, R(x) p) \mathrm{d} p \mathrm{~d} \sigma \mathrm{d} t\right. \\
& =\varepsilon M^{-}+(1-\varepsilon) \int_{0}^{T} \int_{\partial \Omega} \int_{\mathbb{R}^{3}}(v(q) \cdot n(x))_{+} f_{\varepsilon}(t, x, q) \mathrm{dqd} \sigma \mathrm{dt} \\
& =M^{-} .
\end{aligned}
$$

The second uniform estimate is obtained by the conservation of the total energy

$$
W(t)=\frac{1}{2} \int_{\Omega} \int_{\mathbb{R}^{3}} \mathcal{E}(p) f_{\varepsilon}(t, x, p) \mathrm{d} p \mathrm{~d} x+\frac{\varepsilon_{0}}{2} \int_{\Omega}\left(\left|E_{\varepsilon}(t, x)\right|^{2}+c^{2}\left|B_{\varepsilon}(t, x)\right|^{2}\right) \mathrm{d} x .
$$

Since we intend to impose the Silver-Müler boundary condition (7) or the perfect conducting boundary condition (11) we perform the total energy balance for solutions satisfying the boundary condition

$$
n \wedge E_{\varepsilon}+\delta c n \wedge\left(n \wedge B_{\varepsilon}\right)=h(x), \quad(t, x) \in \mathbb{R} \times \partial \Omega .
$$

Thus, when analyzing the Vlasov-Maxwell problem with the Silver-Müler boundary condition (7) we take $\delta=1$ and when studying the Vlasov-Maxwell problem with perfect conducting boundary condition (11) we assume that $h=0$ and let $\delta \searrow 0$.

Proposition 2.2 For any $\varepsilon \in] 0,1]$ the $T$ periodic solution $\left(f_{\varepsilon}, E_{\varepsilon}, B_{\varepsilon}\right)$ satisfies

$\varepsilon \int_{0}^{T} \int_{\partial \Omega} \int_{\mathbb{R}^{3}}(v \cdot n)_{+} \mathcal{E} f_{\varepsilon} \mathrm{d} p \mathrm{~d} \sigma \mathrm{d} t+\frac{\varepsilon_{0} c}{2 \delta} \int_{0}^{T} \int_{\partial \Omega}\left(\left|n \wedge E_{\varepsilon}\right|^{2}+\delta^{2} c^{2}\left|n \wedge B_{\varepsilon}\right|^{2}\right) \mathrm{d} \sigma \mathrm{d} t=\varepsilon K^{-}+\frac{\varepsilon_{0} c}{2 \delta} T H$.

In particular we have for any $\varepsilon \in] 0,1]$

$$
\frac{\varepsilon_{0} c}{2 \delta} \int_{0}^{T} \int_{\partial \Omega}\left(\left|n \wedge E_{\varepsilon}\right|^{2}+\delta^{2} c^{2}\left|n \wedge B_{\varepsilon}\right|^{2}\right) \mathrm{d} \sigma \mathrm{d} t \leq K^{-}+\frac{\varepsilon_{0} c}{2 \delta} T H .
$$

Proof. Multiplying $(3)$ by $\mathcal{E}(p),(5)$ by $\left(E_{\varepsilon}(t, x), c^{2} B_{\varepsilon}(t, x)\right)$ yields after integration $\frac{d}{d t} \int_{\Omega} \int_{\mathbb{R}^{3}} \mathcal{E}(p) f_{\varepsilon} \mathrm{d} p \mathrm{~d} x+\int_{\partial \Omega} \int_{\mathbb{R}^{3}}(v(p) \cdot n(x)) \mathcal{E}(p) f_{\varepsilon} \mathrm{d} p \mathrm{~d} \sigma-q \int_{\Omega} \int_{\mathbb{R}^{3}}\left(v(p) \cdot E_{\varepsilon}\right) f_{\varepsilon} \mathrm{d} p \mathrm{~d} x=0$ and

$$
\begin{aligned}
\frac{d}{d t} \frac{1}{2} \int_{\Omega}\left(\left|E_{\varepsilon}\right|^{2}+c^{2}\left|B_{\varepsilon}\right|^{2}\right) \mathrm{d} x & -c^{2} \int_{\partial \Omega}\left(n(x) \wedge B_{\varepsilon}\right) \cdot E_{\varepsilon} \mathrm{d} \sigma \\
& =-\frac{q}{\varepsilon_{0}} \int_{\Omega} \int_{\mathbb{R}^{3}}\left(v(p) \cdot E_{\varepsilon}\right) f_{\varepsilon} \mathrm{d} p \mathrm{~d} x
\end{aligned}
$$


Putting together the balances for the kinetic and electro-magnetic energies implies

$$
\begin{aligned}
\frac{d}{d t} \int_{\Omega} \int_{\mathbb{R}^{3}} \mathcal{E}(p) f_{\varepsilon} \mathrm{d} p \mathrm{~d} x & +\frac{\varepsilon_{0}}{2} \frac{d}{d t} \int_{\Omega}\left(\left|E_{\varepsilon}\right|^{2}+c^{2}\left|B_{\varepsilon}\right|^{2}\right) \mathrm{d} x+\int_{\partial \Omega} \int_{\mathbb{R}^{3}}(v \cdot n) \mathcal{E}(p) f_{\varepsilon} \mathrm{d} p \mathrm{~d} \sigma \\
& -c^{2} \varepsilon_{0} \int_{\partial \Omega}\left(n \wedge B_{\varepsilon}\right) \cdot E_{\varepsilon} \mathrm{d} \sigma=0 .
\end{aligned}
$$

After integration with respect to $t \in[0, T]$ one gets by time periodicity

$$
\int_{0}^{T} \int_{\partial \Omega} \int_{\mathbb{R}^{3}}(v(p) \cdot n(x)) \mathcal{E}(p) f_{\varepsilon} \mathrm{d} p \mathrm{~d} \sigma \mathrm{d} t-\varepsilon_{0} c^{2} \int_{0}^{T} \int_{\partial \Omega}\left(n \wedge B_{\varepsilon}\right) \cdot E_{\varepsilon} \mathrm{d} \sigma \mathrm{d} t=0
$$

By the Silver-Müler boundary condition (17) we deduce

$$
\begin{aligned}
\delta c \int_{0}^{T} \int_{\partial \Omega}\left(n \wedge B_{\varepsilon}\right) \cdot E_{\varepsilon} \mathrm{d} \sigma \mathrm{d} t & =\delta c \int_{0}^{T} \int_{\partial \Omega}\left(n \wedge\left(n \wedge B_{\varepsilon}\right)\right) \cdot\left(n \wedge E_{\varepsilon}\right) \mathrm{d} \sigma \mathrm{d} t \\
& =\frac{1}{2} \int_{0}^{T} \int_{\partial \Omega}\left(|h|^{2}-\left|n \wedge E_{\varepsilon}\right|^{2}-\delta^{2} c^{2}\left|n \wedge\left(n \wedge B_{\varepsilon}\right)\right|^{2}\right) \mathrm{d} \sigma \mathrm{d} t
\end{aligned}
$$

Using now Lemma 2.1 with the function $F=\mathcal{E}$ we obtain

$$
\begin{aligned}
\int_{0}^{T} \int_{\partial \Omega} \int_{\mathbb{R}^{3}}(v(p) \cdot n(x)) \mathcal{E} f_{\varepsilon} \mathrm{d} p \mathrm{~d} \sigma \mathrm{d} t & =\varepsilon \int_{0}^{T} \int_{\partial \Omega} \int_{\mathbb{R}^{3}}(v(p) \cdot n(x))_{+} \mathcal{E}(p) f_{\mathcal{\varepsilon}} \mathrm{d} p \mathrm{~d} \sigma \mathrm{d} t \\
& -\varepsilon \int_{0}^{T} \int_{\partial \Omega} \int_{\mathbb{R}^{3}}(v(p) \cdot n(x))_{-} \mathcal{E}(p) g \mathrm{~d} p \mathrm{~d} \sigma \mathrm{d} t .
\end{aligned}
$$

Finally combining (18), (19), (20) yields

$$
\begin{aligned}
\varepsilon \int_{0}^{T} \int_{\partial \Omega} \int_{\mathbb{R}^{3}}(v(p) \cdot n(x))_{+} \mathcal{E}(p) f_{\varepsilon} \mathrm{d} p \mathrm{~d} \sigma \mathrm{d} t & +\frac{\varepsilon_{0} c}{2 \delta} \int_{0}^{T} \int_{\partial \Omega}\left(\left|n \wedge E_{\varepsilon}\right|^{2}+\delta^{2} c^{2}\left|n \wedge B_{\varepsilon}\right|^{2}\right) \mathrm{d} \sigma \mathrm{d} t \\
& =\varepsilon \int_{0}^{T} \int_{\partial \Omega} \int_{\mathbb{R}^{3}}(v(p) \cdot n(x))_{-} \mathcal{E}(p) g \mathrm{~d} p \mathrm{~d} \sigma \mathrm{d} t \\
& +\frac{\varepsilon_{0} c}{2 \delta} \int_{0}^{T} \int_{\partial \Omega}|h|^{2} \mathrm{~d} \sigma \mathrm{d} t \\
& =\varepsilon K^{-}+\frac{\varepsilon_{0} c}{2 \delta} T H
\end{aligned}
$$

saying that the tangential traces of the electro-magnetic field are uniformly bounded in $L_{\text {loc }}^{2}\left(\mathbb{R} ; L^{2}(\partial \Omega)^{3}\right)$ with respect to $\left.\left.\varepsilon \in\right] 0,1\right]$.

We need also a uniform bound for the outgoing kinetic energy

$$
\int_{0}^{T} \int_{\partial \Omega} \int_{\mathbb{R}^{3}}(v(p) \cdot n(x))_{+} \mathcal{E}(p) f_{\varepsilon} \mathrm{d} p \mathrm{~d} \sigma \mathrm{d} t
$$

Notice that, for the moment, the equality (21) gives only a bound in $1 / \varepsilon$ (if $\delta>0$ is kept fixed). Actually we will see that (21) provides a uniform bound for the outgoing 
kinetic energy, but this requires the orthogonal decomposition of the tangential field $h \in L^{2}(\partial \Omega)^{3}$ into irrotational and rotational parts. These result is analogous to the well-known orthogonal decompsition result for fields of $L^{2}(\Omega)^{3}$ (see [10] pp. 22). For the sake of the presentation we give here some details cf. [5]. We assume that $\Omega$ is bounded and smooth (generally $C^{1}$ ). For any function $u \in C^{1}(\partial \Omega)$ we denote by $\nabla_{\tau} u$ the tangential gradient of $u$. We also define $\operatorname{curl}_{\tau} u:=n \wedge \nabla_{\tau} u$ if $u \in C^{1}(\partial \Omega)$. It is easily seen that $n \cdot \nabla_{\tau} u=0, n \cdot \operatorname{curl}_{\tau} u=0$ and a direct computation shows that $\nabla_{\tau}$ and $\operatorname{curl}_{\tau}$ are orthogonal in $L^{2}(\partial \Omega)^{3}$

$$
\int_{\partial \Omega} \nabla_{\tau} u \cdot\left(n \wedge \nabla_{\tau} v\right) \mathrm{d} \sigma=0, \quad u, v \in C^{1}(\partial \Omega) .
$$

Moreover, by density we have

$$
\int_{\partial \Omega} \nabla_{\tau} \varphi \cdot\left(n \wedge \nabla_{\tau} \psi\right) \mathrm{d} \sigma=0, \quad \varphi, \psi \in H^{1}(\partial \Omega) .
$$

For the definition of Sobolev spaces on $\partial \Omega$ the reader can refer to [21]. We introduce the notations : $X=\left\{u \in L^{2}(\partial \Omega)^{3} \mid n \cdot u(x)=0\right.$ a.e. $\left.x \in \partial \Omega\right\}, Y=\left\{\nabla_{\tau} \varphi \mid \varphi \in H^{1}(\partial \Omega)\right\}$, $Z=\left\{n \wedge \nabla_{\tau} \psi \mid \psi \in H^{1}(\partial \Omega)\right\}$.

Proposition 2.3 (cf. [5], Proposition A.5, pp. 487) Assume that $\partial \Omega$ is bounded, simply connected and regular $\left(C^{1}\right)$. Then $Y$ and $Z$ are closed orthogonal subspaces of $X$ and we have the decomposition

$$
X=Y+Z
$$

By the previous result we deduce that there are $h_{1}, h_{2} \in H^{1}(\partial \Omega)$ such that

$$
h=\nabla_{\tau} h_{1}+n \wedge \nabla_{\tau} h_{2}
$$

Actually the functions $h_{1}, h_{2}$ are unique up to a constant. Without loss of generality we assume that $\int_{\partial \Omega} h_{1} \mathrm{~d} \sigma=\int_{\partial \Omega} h_{2} \mathrm{~d} \sigma=0$. By the orthogonality we have

$\int_{\partial \Omega}|h|^{2} \mathrm{~d} \sigma=\int_{\partial \Omega}\left|\nabla_{\tau} h_{1}\right|^{2} \mathrm{~d} \sigma+\int_{\partial \Omega}\left|n \wedge \nabla_{\tau} h_{2}\right|^{2} \mathrm{~d} \sigma=\int_{\partial \Omega}\left|\nabla_{\tau} h_{1}\right|^{2} \mathrm{~d} \sigma+\int_{\partial \Omega}\left|\nabla_{\tau} h_{2}\right|^{2} \mathrm{~d} \sigma$ and by Poincaré inequality one gets

$$
\left\|h_{1}\right\|_{H^{1}(\partial \Omega)}+\left\|h_{2}\right\|_{H^{1}(\partial \Omega)} \leq C(\Omega)\|h\|_{L^{2}(\partial \Omega)} .
$$

We recall now the divergence equations verified on the boundary $\mathbb{R} \times \partial \Omega$ by $T$ periodic solutions of the Maxwell equations cf. [5]. We denote by $\nabla_{(t, \tau)}, \operatorname{div}_{(t, \tau)}$ the gradient and divergence operators on $\mathbb{R} \times \partial \Omega$. 
Proposition 2.4 Assume that $\Omega$ is regular and consider $(E, B) \in L_{\text {loc }}^{2}\left(\mathbb{R} ; L^{2}(\Omega)^{3}\right)^{2}$ a $T$ periodic weak solution for the Maxwell equations

$$
\partial_{t} E-c^{2} \operatorname{curl}_{x} B=-\frac{j(t, x)}{\varepsilon_{0}}, \partial_{t} B+\operatorname{curl}_{x} E=0, \operatorname{div}_{x} E=\frac{\rho(t, x)}{\varepsilon_{0}}, \operatorname{div}_{x} B=0
$$

with tangential and normal traces $(n \wedge E, n \wedge B) \in L_{\mathrm{loc}}^{2}\left(\mathbb{R} ; L^{2}(\partial \Omega)^{3}\right)^{2}$, respectively $((n \cdot E),(n \cdot B)) \in L_{\text {loc }}^{2}\left(\mathbb{R} ; L^{2}(\partial \Omega)\right)^{2}$. We assume also that the charge density $\rho$ belongs to $L_{\mathrm{loc}}^{1}\left(\mathbb{R} ; L^{1}(\Omega)\right)$, the current density belongs to $L_{\mathrm{loc}}^{1}\left(\mathbb{R} ; L^{1}(\Omega)^{3}\right)$ and that the continuity equation $\partial_{t} \rho+\operatorname{div}_{x} j=0$ holds true in $\mathcal{D}_{\text {per }}^{\prime}(\mathbb{R} \times \bar{\Omega})$

$\int_{0}^{T} \int_{\Omega} \rho \partial_{t} \varphi \mathrm{d} x \mathrm{~d} t+\int_{0}^{T} \int_{\Omega} j \cdot \nabla_{x} \varphi \mathrm{d} x \mathrm{~d} t=\int_{0}^{T} \int_{\partial \Omega}(n \cdot j) \varphi \mathrm{d} \sigma \mathrm{d} t, \quad \forall \varphi \in C^{1}(\mathbb{R} \times \bar{\Omega}), T$ periodic for some function $(n \cdot j) \in L_{\text {loc }}^{1}\left(\mathbb{R} ; L^{1}(\partial \Omega)\right)$. Then the traces of the electro-magnetic field verify the following divergence equations in $\mathcal{D}_{\text {per }}^{\prime}(\mathbb{R} \times \partial \Omega)$

$$
\operatorname{div}_{(t, \tau)}\left((n \cdot E), c^{2}(n \wedge B)\right)=-\frac{(n \cdot j)}{\varepsilon_{0}}, \operatorname{div}_{(t, \tau)}((n \cdot B),-(n \wedge E))=0
$$

i.e.,

$$
-\int_{0}^{T} \int_{\partial \Omega}(n \cdot E) \partial_{t} \psi \mathrm{d} \sigma \mathrm{d} t-c^{2} \int_{0}^{T} \int_{\partial \Omega}(n \wedge B) \cdot \nabla_{\tau} \psi \mathrm{d} \sigma \mathrm{d} t=-\frac{1}{\varepsilon_{0}} \int_{0}^{T} \int_{\partial \Omega}(n \cdot j) \psi \mathrm{d} \sigma \mathrm{d} t
$$

and

$$
-\int_{0}^{T} \int_{\partial \Omega}(n \cdot B) \partial_{t} \psi \mathrm{d} \sigma \mathrm{d} t+\int_{0}^{T} \int_{\partial \Omega}(n \wedge E) \cdot \nabla_{\tau} \psi \mathrm{d} \sigma \mathrm{d} t=0
$$

for all function $\psi \in C^{1}(\mathbb{R} \times \partial \Omega)$, T periodic.

Proof. Consider the test function $\eta(t) \nabla_{x} \varphi$, where $\eta \in C^{1}(\mathbb{R})$ is $T$ periodic and $\varphi \in C^{1}(\bar{\Omega})$. By using the first equation of (23) with this test function, we deduce

$$
-\int_{0}^{T} \int_{\Omega} \eta^{\prime}(t) E(t, x) \cdot \nabla_{x} \varphi \mathrm{d} x \mathrm{~d} t-c^{2} \int_{0}^{T} \int_{\partial \Omega} \eta(t)(n \wedge B) \cdot \nabla_{x} \varphi \mathrm{d} \sigma \mathrm{d} t=-\frac{1}{\varepsilon_{0}} \int_{0}^{T} \int_{\Omega} \eta(t) j \cdot \nabla_{x} \varphi \mathrm{d} x \mathrm{~d} t .
$$

By using now the third equation of (23) with the test function $-\eta^{\prime}(t) \varphi(x)$ we deduce that

$-\int_{0}^{T} \int_{\partial \Omega} \eta^{\prime}(t)(n \cdot E) \varphi(x) \mathrm{d} \sigma \mathrm{d} t+\int_{0}^{T} \int_{\Omega} \eta^{\prime}(t) E(t, x) \cdot \nabla_{x} \varphi \mathrm{d} x \mathrm{~d} t=-\frac{1}{\varepsilon_{0}} \int_{0}^{T} \int_{\Omega} \rho(t, x) \eta^{\prime}(t) \varphi(x) \mathrm{d} x \mathrm{~d} t$.

By adding the equations (24), (25), by observing that $(n \wedge B) \cdot \nabla_{x} \varphi=(n \wedge B) \cdot \nabla_{\tau} \varphi$ and by using the continuity equation finally we obtain that

$$
-\int_{0}^{T} \int_{\partial \Omega}(n \cdot E) \partial_{t} \psi \mathrm{d} \sigma \mathrm{d} t-c^{2} \int_{0}^{T} \int_{\partial \Omega}(n \wedge B) \cdot \nabla_{\tau} \psi \mathrm{d} \sigma \mathrm{d} t=-\frac{1}{\varepsilon_{0}} \int_{0}^{T} \int_{\partial \Omega}(n \cdot j) \psi \mathrm{d} \sigma \mathrm{d} t,
$$


for all $\psi(t, x)=\eta(t) \varphi(x)$. By density we deduce that the previous equality holds for all test function $\psi \in C^{1}(\mathbb{R} \times \partial \Omega), T$ periodic, or $\operatorname{div}_{(t, \tau)}\left((n \cdot E), c^{2}(n \wedge B)\right)=-\frac{(n \cdot j)}{\varepsilon_{0}}$ in $\mathcal{D}_{p e r}^{\prime}(\mathbb{R} \times \partial \Omega)$. In order to establish the second divergence equation on the boundary we use the second equation of (23) with the test function $\eta(t) \nabla_{x} \varphi$ which gives

$$
-\int_{0}^{T} \int_{\Omega} \eta^{\prime}(t) B(t, x) \cdot \nabla_{x} \varphi \mathrm{d} x \mathrm{~d} t+\int_{0}^{T} \int_{\partial \Omega} \eta(t)(n \wedge E) \cdot \nabla_{x} \varphi \mathrm{d} \sigma \mathrm{d} t=0 .
$$

By using also the fourth equation of (23) one gets finally :

$$
-\int_{0}^{T} \int_{\partial \Omega}(n \cdot B) \partial_{t} \psi \mathrm{d} \sigma \mathrm{d} t+\int_{0}^{T} \int_{\partial \Omega}(n \wedge E) \cdot \nabla_{\tau} \psi \mathrm{d} \sigma \mathrm{d} t=0
$$

or $\operatorname{div}_{(t, \tau)}((n \cdot B),-(n \wedge E))=0$ in $\mathcal{D}_{p e r}^{\prime}(\mathbb{R} \times \partial \Omega)$.

Based on the previous divergence equations satisfied by the electro-magnetic traces, we derive the following representation for the electro-magnetic energy flux $\varepsilon_{0} c^{2} \int_{0}^{T} \int_{\partial \Omega}(n \wedge$ $\left.B_{\varepsilon}\right) \cdot E_{\varepsilon} \mathrm{d} \sigma \mathrm{d} t$

Lemma 2.2 For any $\varepsilon \in] 0,1]$ the $T$ periodic solution $\left(f_{\varepsilon}, E_{\varepsilon}, B_{\varepsilon}\right)$ satisfies

$$
\begin{aligned}
-\int_{0}^{T} \int_{\partial \Omega} \delta c\left(n \wedge B_{\varepsilon}\right) \cdot E_{\varepsilon} \mathrm{d} \sigma \mathrm{d} t & =\frac{1}{2} \int_{0}^{T} \int_{\partial \Omega}\left|n \wedge E_{\varepsilon}-n \wedge \nabla_{\tau} h_{2}\right|^{2} \mathrm{~d} \sigma \mathrm{d} t \\
& +\frac{1}{2} \int_{0}^{T} \int_{\partial \Omega}\left|\delta c n \wedge\left(n \wedge B_{\varepsilon}\right)-\nabla_{\tau} h_{1}\right|^{2} \mathrm{~d} \sigma \mathrm{d} t \\
& -\frac{\delta}{\varepsilon_{0} c} \int_{0}^{T} \int_{\partial \Omega}\left(n \cdot j_{\varepsilon}\right) h_{2} \mathrm{~d} \sigma \mathrm{d} t
\end{aligned}
$$

where $h=\nabla_{\tau} h_{1}+n \wedge \nabla_{\tau} h_{2}$ is the orthogonal decomposition of the tangential field $h \in L^{2}(\partial \Omega)^{3}$ into irrotational and rotational parts.

Proof. By the Silver-Müler boundary condition (17)

$$
n \wedge E_{\varepsilon}+\delta c n \wedge\left(n \wedge B_{\varepsilon}\right)=h=\nabla_{\tau} h_{1}+n \wedge \nabla_{\tau} h_{2}
$$

we have

$$
n \wedge E_{\varepsilon}-n \wedge \nabla_{\tau} h_{2}=-\left(\delta c n \wedge\left(n \wedge B_{\varepsilon}\right)-\nabla_{\tau} h_{1}\right)
$$


and therefore we can write

$$
\begin{aligned}
-\delta c\left(n \wedge\left(n \wedge B_{\varepsilon}\right)\right) \cdot\left(n \wedge E_{\varepsilon}\right)= & -\left(\delta c\left(n \wedge\left(n \wedge B_{\varepsilon}\right)\right)-\nabla_{\tau} h_{1}+\nabla_{\tau} h_{1}\right) \\
& \cdot\left(n \wedge E_{\varepsilon}-n \wedge \nabla_{\tau} h_{2}+n \wedge \nabla_{\tau} h_{2}\right) \\
= & \frac{1}{2}\left|n \wedge E_{\varepsilon}-n \wedge \nabla_{\tau} h_{2}\right|^{2}+\frac{1}{2}\left|\delta c n \wedge\left(n \wedge B_{\varepsilon}\right)-\nabla_{\tau} h_{1}\right|^{2} \\
& -\nabla_{\tau} h_{1} \cdot\left(n \wedge \nabla_{\tau} h_{2}\right)-\left(n \wedge E_{\varepsilon}-n \wedge \nabla_{\tau} h_{2}\right) \cdot \nabla_{\tau} h_{1} \\
- & \left(\delta c n \wedge\left(n \wedge B_{\varepsilon}\right)-\nabla_{\tau} h_{1}\right) \cdot\left(n \wedge \nabla_{\tau} h_{2}\right) .
\end{aligned}
$$

We will transform the last three terms. By the orthogonality of $\nabla_{\tau}$ and $n \wedge \nabla_{\tau}$ we have

$$
\begin{gathered}
\int_{\partial \Omega} \nabla_{\tau} h_{1} \cdot\left(n \wedge \nabla_{\tau} h_{2}\right) \mathrm{d} \sigma=0 \\
\int_{\partial \Omega}\left(n \wedge E_{\varepsilon}-n \wedge \nabla_{\tau} h_{2}\right) \cdot \nabla_{\tau} h_{1} \mathrm{~d} \sigma=\int_{\partial \Omega}\left(n \wedge E_{\varepsilon}\right) \cdot \nabla_{\tau} h_{1} \mathrm{~d} \sigma \\
\int_{\partial \Omega}\left(\delta c n \wedge\left(n \wedge B_{\varepsilon}\right)-\nabla_{\tau} h_{1}\right) \cdot\left(n \wedge \nabla_{\tau} h_{2}\right) \mathrm{d} \sigma \\
=\int_{\partial \Omega} \delta c\left(n \wedge\left(n \wedge B_{\varepsilon}\right)\right) \cdot\left(n \wedge \nabla_{\tau} h_{2}\right) \mathrm{d} \sigma \\
=\int_{\partial \Omega} \delta c\left(n \wedge B_{\varepsilon}\right) \cdot \nabla_{\tau} h_{2} \mathrm{~d} \sigma
\end{gathered}
$$

implying that

$$
\begin{aligned}
-\delta c \int_{0}^{T} \int_{\partial \Omega}\left(n \wedge B_{\varepsilon}\right) \cdot E_{\varepsilon} \mathrm{d} \sigma \mathrm{d} t & =\frac{1}{2} \int_{0}^{T} \int_{\partial \Omega}\left|n \wedge E_{\varepsilon}-n \wedge \nabla_{\tau} h_{2}\right|^{2} \mathrm{~d} \sigma \mathrm{d} t \\
& +\frac{1}{2} \int_{0}^{T} \int_{\partial \Omega}\left|\delta c n \wedge\left(n \wedge B_{\varepsilon}\right)-\nabla_{\tau} h_{1}\right|^{2} \mathrm{~d} \sigma \mathrm{d} t \\
& -\int_{0}^{T} \int_{\partial \Omega}\left(n \wedge E_{\varepsilon}\right) \cdot \nabla_{\tau} h_{1} \mathrm{~d} \sigma \mathrm{d} t \\
& -\delta c \int_{0}^{T} \int_{\partial \Omega}\left(n \wedge B_{\varepsilon}\right) \cdot \nabla_{\tau} h_{2} \mathrm{~d} \sigma \mathrm{d} t .
\end{aligned}
$$

Applying now the divergence equations in the conclusion of Proposition 2.4 with the test function $h_{2}(x), h_{1}(x)$ yields

$$
-c^{2} \int_{0}^{T} \int_{\partial \Omega}\left(n \wedge B_{\varepsilon}\right) \cdot \nabla_{\tau} h_{2} \mathrm{~d} \sigma \mathrm{d} t=-\frac{1}{\varepsilon_{0}} \int_{0}^{T} \int_{\partial \Omega}\left(n \cdot j_{\varepsilon}\right) h_{2} \mathrm{~d} \sigma \mathrm{d} t
$$

and

$$
\int_{0}^{T} \int_{\partial \Omega}\left(n \wedge E_{\varepsilon}\right) \cdot \nabla_{\tau} h_{1} \mathrm{~d} \sigma \mathrm{d} t=0
$$


Finally one gets by (28)

$$
\begin{aligned}
-\delta c \int_{0}^{T} \int_{\partial \Omega}\left(n \wedge B_{\varepsilon}\right) \cdot E_{\varepsilon} \mathrm{d} \sigma \mathrm{d} t & =\frac{1}{2} \int_{0}^{T} \int_{\partial \Omega}\left|n \wedge E_{\varepsilon}-n \wedge \nabla_{\tau} h_{2}\right|^{2} \mathrm{~d} \sigma \mathrm{d} t \\
& +\frac{1}{2} \int_{0}^{T} \int_{\partial \Omega}\left|\delta c n \wedge\left(n \wedge B_{\varepsilon}\right)-\nabla_{\tau} h_{1}\right|^{2} \mathrm{~d} \sigma \mathrm{d} t \\
& -\frac{\delta}{\varepsilon_{0} c} \int_{0}^{T} \int_{\partial \Omega}\left(n \cdot j_{\varepsilon}\right) h_{2} \mathrm{~d} \sigma \mathrm{d} t .
\end{aligned}
$$

We derive now a uniform estimate for the outgoing/incoming kinetic energy fluxes $\int_{0}^{T} \int_{\partial \Omega} \int_{\mathbb{R}^{3}}(v(p) \cdot n(x))_{ \pm} \mathcal{E}(p) f_{\varepsilon} \mathrm{d} p \mathrm{~d} \sigma \mathrm{d} t$. This bound is a consequence of the total energy balance in Proposition 2.2 and the representation formula in Lemma 2.2. First we establish a uniform $L^{\infty}$ bound for $\left(f_{\varepsilon}\right)_{\varepsilon}$.

Proposition 2.5 Assume that $g \in L^{\infty}\left(\mathbb{R} \times \Sigma^{-}\right)$is non negative. Then we have for any $\varepsilon \in] 0,1]$

$$
\max \left\{\left\|f_{\varepsilon}\right\|_{L^{\infty}\left(\mathbb{R} \times \Omega \times \mathbb{R}^{3}\right)},\left\|f_{\varepsilon}\right\|_{L^{\infty}\left(\mathbb{R} \times \Sigma^{ \pm}\right)}\right\} \leq\|g\|_{L^{\infty}\left(\mathbb{R} \times \Sigma^{-}\right)} .
$$

Proof. For any fixed $\alpha>0, \varepsilon \in] 0,1]$ and given electro-magnetic field $\left(E_{\varepsilon}, B_{\varepsilon}\right)$ the solution of the Vlasov equation in (9) with the boundary condition (12) is obtained by the iterative scheme

$$
\begin{cases}\alpha f^{(0)}+\partial_{t} f^{(0)}+v(p) \cdot \nabla_{x} f^{(0)}+q\left(E_{\varepsilon}+v(p) \wedge B_{\varepsilon}\right) \cdot \nabla_{p} f^{(0)}=0, & (t, x, p) \in \mathbb{R} \times \Omega \times \mathbb{R}^{3} \\ f^{(0)}(t, x, p)=\varepsilon g(t, x, p), & (t, x, p) \in \mathbb{R} \times \Sigma^{-}\end{cases}
$$

and for any $n \in \mathbb{N}$

$$
\begin{cases}\alpha f^{(n+1)}+\partial_{t} f^{(n+1)}+v \cdot \nabla_{x} f^{(n+1)}+q\left(E_{\varepsilon}+v \wedge B_{\varepsilon}\right) \cdot \nabla_{p} f^{(n+1)}=0, & (t, x, p) \in \mathbb{R} \times \Omega \times \mathbb{R}^{3} \\ f^{(n+1)}(t, x, p)=\varepsilon g(t, x, p)+(1-\varepsilon) f^{(n)}(t, x, R(x) p), & (t, x, p) \in \mathbb{R} \times \Sigma^{-} .\end{cases}
$$

Indeed, we have $0 \leq f^{(0)} \leq f^{(1)}$ on $\mathbb{R} \times \Sigma^{-}$and thus, by comparison principle (which holds true for time periodic solutions and any $\alpha>0$ ) one gets $0 \leq f^{(0)} \leq f^{(1)}$ on $\mathbb{R} \times \Omega \times \mathbb{R}^{3}$ and on $\mathbb{R} \times \Sigma^{+}$. Assuming that $0 \leq f^{(n)} \leq f^{(n+1)}$ on $\mathbb{R} \times \Omega \times \mathbb{R}^{3}$ and $\mathbb{R} \times \Sigma^{+}$ we deduce that $f^{(n+1)} \leq f^{(n+2)}$ on $\mathbb{R} \times \Sigma^{-}$, which implies by comparison principle that $f^{(n+1)} \leq f^{(n+2)}$ on $\mathbb{R} \times \Omega \times \mathbb{R}^{3}$ and on $\mathbb{R} \times \Sigma^{+}$. Finally we check easily that the monotonous sequence $\left(f^{(n)}\right)_{n}$ converges to a $T$ periodic solution $f_{\varepsilon, \alpha}$ of the Vlasov equation in (9) satisfying the boundary condition

$$
f_{\varepsilon, \alpha}(t, x, p)=\varepsilon g(t, x, p)+(1-\varepsilon) f_{\varepsilon, \alpha}(t, x, R(x) p), \quad(t, x, p) \in \mathbb{R} \times \Sigma^{-} .
$$


Obviously we have

$$
\max \left\{\left\|f^{(0)}\right\|_{L^{\infty}\left(\mathbb{R} \times \Omega \times \mathbb{R}^{3}\right)},\left\|f^{(0)}\right\|_{L^{\infty}\left(\mathbb{R} \times \Sigma^{ \pm}\right)}\right\} \leq\|g\|_{L^{\infty}\left(\mathbb{R} \times \Sigma^{-}\right)} .
$$

Assuming that

$$
\max \left\{\left\|f^{(n)}\right\|_{L^{\infty}\left(\mathbb{R} \times \Omega \times \mathbb{R}^{3}\right)},\left\|f^{(n)}\right\|_{L^{\infty}\left(\mathbb{R} \times \Sigma^{ \pm}\right)}\right\} \leq\|g\|_{L^{\infty}\left(\mathbb{R} \times \Sigma^{-}\right)}
$$

implies that $\left\|f^{(n+1)}\right\|_{L^{\infty}\left(\mathbb{R} \times \Sigma^{-}\right)} \leq\|g\|_{L^{\infty}\left(\mathbb{R} \times \Sigma^{-}\right)}$and therefore

$$
\max \left\{\left\|f^{(n+1)}\right\|_{L^{\infty}\left(\mathbb{R} \times \Omega \times \mathbb{R}^{3}\right)},\left\|f^{(n+1)}\right\|_{L^{\infty}\left(\mathbb{R} \times \Sigma^{+}\right)}\right\} \leq\|g\|_{L^{\infty}\left(\mathbb{R} \times \Sigma^{-}\right)} .
$$

Passing to the limit with respect to $n$ one gets

$$
\max \left\{\left\|f_{\varepsilon, \alpha}\right\|_{L^{\infty}\left(\mathbb{R} \times \Omega \times \mathbb{R}^{3}\right)},\left\|f_{\varepsilon, \alpha}\right\|_{L^{\infty}\left(\mathbb{R} \times \Sigma^{ \pm}\right)}\right\} \leq\|g\|_{L^{\infty}\left(\mathbb{R} \times \Sigma^{-}\right)} .
$$

In order to pass to the limit for $\alpha \searrow 0$ observe that if $0<\alpha \leq \beta$ then $f_{\varepsilon, \alpha} \geq f_{\varepsilon, \beta}$ (actually this inequality holds true for any elements $f_{\alpha}^{(n)}, f_{\beta}^{(n)}$ in the iterative schemes associated to the parameters $\alpha, \beta)$. Finally it is easily seen that for any $\varepsilon \in] 0,1]$ the function $f_{\varepsilon}=\lim _{\alpha \searrow 0} f_{\varepsilon, \alpha}$ solves the problem (3), (12) and satisfies

$$
\max \left\{\left\|f_{\varepsilon}\right\|_{L^{\infty}\left(\mathbb{R} \times \Omega \times \mathbb{R}^{3}\right)},\left\|f_{\varepsilon}\right\|_{L^{\infty}\left(\mathbb{R} \times \Sigma^{ \pm}\right)}\right\} \leq\|g\|_{L^{\infty}\left(\mathbb{R} \times \Sigma^{-}\right)} .
$$

Proposition 2.6 There is a constant $C$ depending on $T, \Omega,\|h\|_{L^{2}(\partial \Omega)},\|g\|_{L^{\infty}\left(\mathbb{R} \times \Sigma^{-}\right)}$, $M^{-}, K^{-}$such that for any $\left.\left.\varepsilon \in\right] 0,1\right]$ the $T$ periodic solution $\left(f_{\varepsilon}, E_{\varepsilon}, B_{\varepsilon}\right)$ satisfies

$$
\begin{gathered}
\int_{0}^{T} \int_{\partial \Omega} \int_{\mathbb{R}^{3}}(v(p) \cdot n(x))_{ \pm} \mathcal{E}(p) f_{\varepsilon}(t, x, p) \mathrm{d} p \mathrm{~d} \sigma \mathrm{d} t \leq C \\
\frac{\varepsilon_{0} c}{2 \delta} \int_{0}^{T} \int_{\partial \Omega}\left|n \wedge E_{\varepsilon}-n \wedge \nabla_{\tau} h_{2}\right|^{2}+\left|\delta c\left(n \wedge\left(n \wedge B_{\varepsilon}\right)\right)-\nabla_{\tau} h_{1}\right|^{2} \mathrm{~d} \sigma \mathrm{d} t \leq C \varepsilon .
\end{gathered}
$$

Proof. Combining (18), (20), (26) yields

$$
\begin{aligned}
\varepsilon \int_{0}^{T} \int_{\partial \Omega} \int_{\mathbb{R}^{3}}(v(p) \cdot n(x))_{+} \mathcal{E}(p) f_{\varepsilon} \mathrm{d} p \mathrm{~d} \sigma \mathrm{d} t & +\frac{\varepsilon_{0} c}{2 \delta} \int_{0}^{T} \int_{\partial \Omega}\left|n \wedge E_{\varepsilon}-n \wedge \nabla_{\tau} h_{2}\right|^{2} \mathrm{~d} \sigma \mathrm{d} t \\
& +\frac{\varepsilon_{0} c}{2 \delta} \int_{0}^{T} \int_{\partial \Omega}\left|\delta c\left(n \wedge\left(n \wedge B_{\varepsilon}\right)\right)-\nabla_{\tau} h_{1}\right|^{2} \mathrm{~d} \sigma \mathrm{d} t \\
& =\varepsilon \int_{0}^{T} \int_{\partial \Omega} \int_{\mathbb{R}^{3}}(v(p) \cdot n(x))_{-} \mathcal{E}(p) g \mathrm{~d} p \mathrm{~d} \sigma \mathrm{d} t \\
& +\int_{0}^{T} \int_{\partial \Omega}\left(n \cdot j_{\varepsilon}\right) h_{2} \mathrm{~d} \sigma \mathrm{d} t .
\end{aligned}
$$


Using now Lemma 2.1 with $F=1$ implies

$$
\begin{aligned}
n(x) \cdot j_{\varepsilon}(t, x)=\int_{\mathbb{R}^{3}}(v(p) \cdot n(x)) f_{\varepsilon} \mathrm{d} p & =\varepsilon \int_{\mathbb{R}^{3}}(v(p) \cdot n(x))_{+} f_{\varepsilon} \mathrm{d} p \\
& -\varepsilon \int_{\mathbb{R}^{3}}(v(p) \cdot n(x))_{-} g \mathrm{~d} p
\end{aligned}
$$

and therefore

$$
\begin{aligned}
\int_{0}^{T} \int_{\partial \Omega}\left(n \cdot j_{\varepsilon}\right) h_{2} \mathrm{~d} \sigma \mathrm{d} t & =\varepsilon \int_{0}^{T} \int_{\partial \Omega} \int_{\mathbb{R}^{3}}(v(p) \cdot n(x))_{+} h_{2} f_{\varepsilon} \mathrm{d} p \mathrm{~d} \sigma \mathrm{d} t \\
& -\varepsilon \int_{0}^{T} \int_{\partial \Omega} \int_{\mathbb{R}^{3}}(v(p) \cdot n(x))_{-} h_{2} g \mathrm{~d} p \mathrm{~d} \sigma \mathrm{d} t .
\end{aligned}
$$

Putting together (29), (31) one gets

$$
\begin{aligned}
\int_{0}^{T} \int_{\partial \Omega} \int_{\mathbb{R}^{3}}(v(p) \cdot n(x))_{+} \mathcal{E}(p) f_{\varepsilon} \mathrm{d} p \mathrm{~d} \sigma \mathrm{d} t & +\frac{\varepsilon_{0} c}{2 \varepsilon \delta} \int_{0}^{T} \int_{\partial \Omega}\left|n \wedge E_{\varepsilon}-n \wedge \nabla_{\tau} h_{2}\right|^{2} \mathrm{~d} \sigma \mathrm{d} t \\
& +\frac{\varepsilon_{0} c}{2 \varepsilon \delta} \int_{0}^{T} \int_{\partial \Omega}\left|\delta c\left(n \wedge\left(n \wedge B_{\varepsilon}\right)\right)-\nabla_{\tau} h_{1}\right|^{2} \mathrm{~d} \sigma \mathrm{d} t \\
& =\int_{0}^{T} \int_{\partial \Omega} \int_{\mathbb{R}^{3}}(v(p) \cdot n(x))_{-}\left(\mathcal{E}(p)-h_{2}(x)\right) g \mathrm{~d} p \mathrm{~d} \sigma \mathrm{d} t \\
& +\int_{0}^{T} \int_{\partial \Omega} \int_{\mathbb{R}^{3}}(v(p) \cdot n(x))_{+} h_{2} f_{\varepsilon} \mathrm{d} p \mathrm{~d} \sigma \mathrm{d} t .
\end{aligned}
$$

We estimate now $\int_{0}^{T} \int_{\partial \Omega} \int_{\mathbb{R}^{3}}(v(p) \cdot n(x))_{+} h_{2} f_{\varepsilon} \mathrm{d} p \mathrm{~d} \sigma \mathrm{d} t$ and $\int_{0}^{T} \int_{\partial \Omega} \int_{\mathbb{R}^{3}}(v(p) \cdot n(x))_{-} h_{2} g \mathrm{~d} p \mathrm{~d} \sigma \mathrm{d} t$ by using Sobolev and interpolation inequalities. Since $\left(f_{\varepsilon}\right)_{0<\varepsilon \leq 1}$ is uniformly bounded we have for a.a. $(t, x) \in[0, T] \times \partial \Omega$

$$
\begin{aligned}
\int_{\mathbb{R}^{3}}(v(p) \cdot n(x))_{+} f_{\varepsilon} \mathrm{d} p & =\int_{\mathbb{R}^{3}}(v(p) \cdot n(x))_{+} f_{\varepsilon} \mathbf{1}_{\{|p|<R\}} \mathrm{d} p \\
& +\int_{\mathbb{R}^{3}}(v(p) \cdot n(x))_{+} f_{\varepsilon} \mathbf{1}_{\{|p| \geq R\}} \mathrm{d} p \\
& \leq C R^{4}\|g\|_{L^{\infty}}+\frac{1}{C R} \int_{\mathbb{R}^{3}}(v(p) \cdot n(x))_{+}(1+\mathcal{E}(p)) f_{\varepsilon} \mathrm{d} p
\end{aligned}
$$

By taking the optimal value for $R$ one gets

$$
\int_{\mathbb{R}^{3}}(v(p) \cdot n(x))_{+} f_{\varepsilon} \mathrm{d} p \leq C\|g\|_{L^{\infty}}^{1 / 5}\left(\int_{\mathbb{R}^{3}}(v(p) \cdot n(x))_{+}(1+\mathcal{E}(p)) f_{\varepsilon} \mathrm{d} p\right)^{4 / 5}
$$

implying that

$$
\begin{aligned}
\left\|\int_{\mathbb{R}^{3}}(v \cdot n)_{+} f_{\varepsilon}(\cdot, \cdot, p) \mathrm{d} p\right\|_{L^{\frac{5}{4}(] 0, T[\times \partial \Omega)}} & \leq C\|g\|_{L^{\infty}}^{1 / 5}\left(\int_{0}^{T} \int_{\partial \Omega} \int_{\mathbb{R}^{3}}(v \cdot n)_{+}(1+\mathcal{E}) f_{\mathcal{\varepsilon}} \mathrm{d} p \mathrm{~d} \sigma \mathrm{d} t\right)^{4 / 5} \\
& \leq C\|g\|_{L^{\infty}}^{1 / 5}\left(\int_{0}^{T} \int_{\partial \Omega} \int_{\mathbb{R}^{3}}(v \cdot n)_{+} \mathcal{E} f_{\varepsilon} \mathrm{d} p \mathrm{~d} \sigma \mathrm{d} t+M^{-}\right)^{4 / 5}(33)
\end{aligned}
$$


Notice that in the last inequality we have used Proposition 2.1. Similarly one gets

$$
\begin{aligned}
\left\|\int_{\mathbb{R}^{3}}(v \cdot n)_{-} g(\cdot, \cdot, p) \mathrm{d} p\right\|_{L^{\frac{5}{4}(] 0, T[\times \partial \Omega)}} & \leq C\|g\|_{L^{\infty}}^{1 / 5}\left(\int_{0}^{T} \int_{\partial \Omega} \int_{\mathbb{R}^{3}}(v \cdot n)_{-}(1+\mathcal{E}) g \mathrm{~d} p \mathrm{~d} \sigma \mathrm{d} t\right)^{4 / 5} \\
& \leq C\|g\|_{L^{\infty}}^{1 / 5}\left(M^{-}+K^{-}\right)^{4 / 5}
\end{aligned}
$$

Using now the Sobolev inclusion $H^{1}(\partial \Omega) \rightarrow L^{5}(\partial \Omega)$ and the Hölder inequality we obtain

$$
\begin{aligned}
\left|\int_{0}^{T} \int_{\partial \Omega} \int_{\mathbb{R}^{3}}(v \cdot n)_{+} h_{2} f_{\varepsilon} \mathrm{d} p \mathrm{~d} \sigma \mathrm{d} t\right| & \leq\left\|h_{2}\right\|_{L^{5}(] 0, T[\times \partial \Omega)}\left\|\int_{\mathbb{R}^{3}}(v \cdot n)_{+} f_{\varepsilon} \mathrm{d} p\right\|_{L^{\frac{5}{4}(] 0, T[\times \partial \Omega)}} \\
& \leq C(\Omega, T)\left\|h_{2}\right\|_{H^{1}(\partial \Omega)}\left\|\int_{\mathbb{R}^{3}}(v \cdot n)_{+} f_{\varepsilon} \mathrm{d} p\right\|_{L^{\frac{5}{4}(] 0, T[\times \partial \Omega)}} \\
& \leq C(\Omega, T)\|h\|_{L^{2}(\partial \Omega)}\|g\|_{L^{\infty}}^{1 / 5} \\
& \times\left(M^{-}+\int_{0}^{T} \int_{\partial \Omega} \int_{\mathbb{R}^{3}}(v \cdot n)_{+} \mathcal{E}(p) f_{\varepsilon} \mathrm{d} p \mathrm{~d} \sigma \mathrm{d} t\right)^{4 / 5} .(35)
\end{aligned}
$$

In the same manner we have

$$
\left|\int_{0}^{T} \int_{\partial \Omega} \int_{\mathbb{R}^{3}}(v \cdot n)_{-} h_{2} g \mathrm{~d} p \mathrm{~d} \sigma \mathrm{d} t\right| \leq C(\Omega, T)\|h\|_{L^{2}(\partial \Omega)}\|g\|_{L^{\infty}}^{1 / 5}\left(M^{-}+K^{-}\right)^{4 / 5} .
$$

Combining (32), (35), (36) clearly gives a uniform bound for the outgoing kinetic energies

$$
\sup _{0<\varepsilon \leq 1} \int_{0}^{T} \int_{\partial \Omega} \int_{\mathbb{R}^{3}}(v(p) \cdot n(x))_{+} \mathcal{E}(p) f_{\varepsilon} \mathrm{d} p \mathrm{~d} \sigma \mathrm{d} t \leq C\left(\Omega, T,\|h\|_{L^{2}},\|g\|_{L^{\infty}}, M^{-}, K^{-}\right) .
$$

Using now the boundary condition $f_{\varepsilon}=\varepsilon g+(1-\varepsilon) f_{\varepsilon}(t, x, R(x) p),(t, x, p) \in \mathbb{R} \times \Sigma^{-}$ also gives a uniform bound for the incoming kinetic energies on $\mathbb{R} \times \Sigma^{-}$. Notice also that (32) implies

$\frac{\varepsilon_{0} c}{2 \delta} \int_{0}^{T} \int_{\partial \Omega}\left|n \wedge E_{\varepsilon}-n \wedge \nabla_{\tau} h_{2}\right|^{2}+\left|\delta c n \wedge\left(n \wedge B_{\varepsilon}\right)-\nabla_{\tau} h_{1}\right|^{2} \mathrm{~d} \sigma \mathrm{d} t \leq C \varepsilon, \quad 0<\varepsilon \leq 1$.

Once we have estimated the tangential traces of the electro-magnetic field, cf. Proposition 2.2 and the kinetic energy flux, cf. Proposition 2.6 it is possible to obtain uniform bounds for the total kinetic and electro-magnetic energy by appealing to the multiplier method [4], [5]. Using the momentum conservation yields 
Proposition 2.7 Assume that $\Omega$ is bounded and strictly star-shaped. Then for any $\varepsilon \in] 0,1]$ we have

$$
\begin{aligned}
& \int_{0}^{T} \int_{\Omega} \int_{\mathbb{R}^{3}} \mathcal{E} f_{\varepsilon} \mathrm{d} p \mathrm{~d} x \mathrm{~d} t+\frac{\varepsilon_{0}}{2} \int_{0}^{T} \int_{\Omega}\left(\left|E_{\varepsilon}\right|^{2}+c^{2}\left|B_{\varepsilon}\right|^{2}\right) \mathrm{d} x \mathrm{~d} t+\frac{\varepsilon_{0}}{2} \int_{0}^{T} \int_{\partial \Omega}\left(n \cdot E_{\varepsilon}\right)^{2}+c^{2}\left(n \cdot B_{\varepsilon}\right)^{2} \mathrm{~d} \sigma \mathrm{d} t \\
\leq & C(\Omega)\left\{\int_{0}^{T} \int_{\partial \Omega} \int_{\mathbb{R}^{3}}|(v(p) \cdot n(x))||p| f_{\varepsilon} \mathrm{d} p \mathrm{~d} \sigma \mathrm{d} t+\frac{\varepsilon_{0}}{2} \int_{0}^{T} \int_{\partial \Omega}\left|n \wedge E_{\varepsilon}\right|^{2}+c^{2}\left|n \wedge B_{\varepsilon}\right|^{2} \mathrm{~d} \sigma \mathrm{d} t\right\} .
\end{aligned}
$$

Moreover, if $\delta=1$, then there is a constant $C$ depending on $\Omega, T,\|h\|_{L^{2}},\|g\|_{L^{\infty}}$, $M^{-}, K^{-}$such that

$$
\begin{gathered}
\int_{0}^{T} \int_{\Omega} \int_{\mathbb{R}^{3}} \mathcal{E}(p) f_{\varepsilon} \mathrm{d} p \mathrm{~d} x \mathrm{~d} t+\frac{\varepsilon_{0}}{2} \int_{0}^{T} \int_{\Omega}\left(\left|E_{\varepsilon}\right|^{2}+c^{2}\left|B_{\varepsilon}\right|^{2}\right) \mathrm{d} x \mathrm{~d} t \leq C \\
\frac{\varepsilon_{0}}{2} \int_{0}^{T} \int_{\partial \Omega}\left(n \cdot E_{\varepsilon}\right)^{2}+c^{2}\left(n \cdot B_{\varepsilon}\right)^{2} \mathrm{~d} \sigma \mathrm{d} t \leq C .
\end{gathered}
$$

Proof. Without loss of generality we assume that $\partial \Omega$ is strictly star-shaped with respect to the origin $0 \in \Omega$

$$
\exists r>0: r \leq(x \cdot n(x)), \quad x \in \partial \Omega \text {. }
$$

We consider $R>0$ such that $\Omega \subset B(0, R)$. The momentum conservation reads

$$
\partial_{t} \int_{\mathbb{R}^{3}} p f_{\varepsilon} \mathrm{d} p+\operatorname{div}_{x} \int_{\mathbb{R}^{3}} p \otimes v(p) f_{\varepsilon} \mathrm{d} p-\left(\rho_{\varepsilon} E_{\varepsilon}+j_{\varepsilon} \wedge B_{\varepsilon}\right)=0
$$

and direct computations with the Maxwell equations yield

$$
\begin{aligned}
\rho_{\varepsilon} E_{\varepsilon}+j_{\varepsilon} \wedge B_{\varepsilon} & =\varepsilon_{0}\left(E_{\varepsilon} \operatorname{div}_{x} E_{\varepsilon}-E_{\varepsilon} \wedge \operatorname{curl}_{x} E_{\varepsilon}\right)+\varepsilon_{0} c^{2}\left(B_{\varepsilon} \operatorname{div}_{x} B_{\varepsilon}-B_{\varepsilon} \wedge \operatorname{curl}_{x} B_{\varepsilon}\right) \\
& -\varepsilon_{0} \partial_{t}\left(E_{\varepsilon} \wedge B_{\varepsilon}\right) .
\end{aligned}
$$

Using the identity

$$
u_{i} \operatorname{div}_{x} u-\left(u \wedge \operatorname{curl}_{x} u\right)_{i}=\sum_{j=1}^{3} \frac{\partial}{\partial x_{j}}\left(u_{i} u_{j}\right)-\frac{1}{2} \frac{\partial}{\partial x_{i}}|u|^{2}, 1 \leq i \leq 3
$$

and the decomposition

$$
\left(E_{\varepsilon}, B_{\varepsilon}\right)=\left(\left(n \cdot E_{\varepsilon}\right) n-n \wedge\left(n \wedge E_{\varepsilon}\right),\left(n \cdot B_{\varepsilon}\right) n-n \wedge\left(n \wedge B_{\varepsilon}\right)\right)
$$


one gets after integration by parts

$$
\begin{aligned}
& \int_{0}^{T} \int_{\Omega}\left(\rho_{\varepsilon} E_{\varepsilon}+j_{\varepsilon} \wedge B_{\varepsilon}\right) \cdot x=-\varepsilon_{0} \int_{0}^{T} \int_{\partial \Omega}\left\{\left(n \cdot E_{\varepsilon}\right)\left(n \wedge\left(n \wedge E_{\varepsilon}\right)\right)+c^{2}\left(n \cdot B_{\varepsilon}\right)\left(n \wedge\left(n \wedge B_{\varepsilon}\right)\right)\right\} \cdot x \\
& +\frac{\varepsilon_{0}}{2} \int_{0}^{T} \int_{\partial \Omega}\left\{\left(n \cdot E_{\varepsilon}\right)^{2}+c^{2}\left(n \cdot B_{\varepsilon}\right)^{2}\right\}(n \cdot x) \mathrm{d} \sigma \mathrm{d} t-\frac{\varepsilon_{0}}{2} \int_{0}^{T} \int_{\partial \Omega}\left\{\left|n \wedge E_{\varepsilon}\right|^{2}+c^{2}\left|n \wedge B_{\varepsilon}\right|^{2}\right\}(n \cdot x) \mathrm{d} \sigma \mathrm{d} t \\
& +\frac{\varepsilon_{0}}{2} \int_{0}^{T} \int_{\Omega}\left\{\left|E_{\varepsilon}\right|^{2}+c^{2}\left|B_{\varepsilon}\right|^{2}\right\} \mathrm{d} x \mathrm{~d} t
\end{aligned}
$$

Multiplying the momentum conservation (40) by $x$ and integrating over $[0, T] \times \Omega$ we obtain

$$
\begin{aligned}
\int_{0}^{T} \int_{\partial \Omega} \int_{\mathbb{R}^{3}}(v(p) \cdot n(x))(p \cdot x) f_{\varepsilon} \mathrm{d} p \mathrm{~d} \sigma \mathrm{d} t & =\int_{0}^{T} \int_{\Omega} \int_{\mathbb{R}^{3}}(v(p) \cdot p) f_{\varepsilon} \mathrm{d} p \mathrm{~d} x \mathrm{~d} t \\
& +\int_{0}^{T} \int_{\Omega}\left(\rho_{\varepsilon} E_{\varepsilon}+j_{\varepsilon} \wedge B_{\varepsilon}\right) \cdot x \mathrm{~d} x \mathrm{~d} t
\end{aligned}
$$

Combining (42), (43) and observing that $\mathcal{E}(p) \leq(v(p) \cdot p)$ yields

$$
\begin{aligned}
& \int_{0}^{T} \int_{\Omega} \int_{\mathbb{R}^{3}} \mathcal{E}(p) f_{\varepsilon} \mathrm{d} p \mathrm{~d} x \mathrm{~d} t+\frac{\varepsilon_{0}}{2} \int_{0}^{T} \int_{\Omega}\left|E_{\varepsilon}\right|^{2}+c^{2}\left|B_{\varepsilon}\right|^{2} \mathrm{~d} x \mathrm{~d} t+\frac{r \varepsilon_{0}}{2} \int_{0}^{T} \int_{\partial \Omega}\left(n \cdot E_{\varepsilon}\right)^{2}+c^{2}\left(n \cdot B_{\varepsilon}\right)^{2} \mathrm{~d} \sigma \mathrm{d} t \\
& \leq R \int_{0}^{T} \int_{\Sigma}|(v(p) \cdot n(x))||p| f_{\varepsilon} \mathrm{d} p \mathrm{~d} \sigma \mathrm{d} t+\frac{R \varepsilon_{0}}{2} \int_{0}^{T} \int_{\partial \Omega}\left\{\left|n \wedge E_{\varepsilon}\right|^{2}+c^{2}\left|n \wedge B_{\varepsilon}\right|^{2}\right\} \mathrm{d} \sigma \mathrm{d} t \\
& +R \varepsilon_{0} \int_{0}^{T} \int_{\partial \Omega}\left\{\left|\left(n \cdot E_{\varepsilon}\right)\right| \cdot\left|n \wedge E_{\varepsilon}\right|+c^{2}\left|\left(n \cdot B_{\varepsilon}\right)\right| \cdot\left|n \wedge B_{\varepsilon}\right|\right\} \mathrm{d} \sigma \mathrm{d} t
\end{aligned}
$$

We obtain (37) by writing

$$
\begin{aligned}
& \varepsilon_{0} \int_{0}^{T} \int_{\partial \Omega}\left|\left(n \cdot E_{\varepsilon}\right)\right|\left|n \wedge E_{\varepsilon}\right| \mathrm{d} \sigma \mathrm{d} t \leq \frac{\mu \varepsilon_{0}}{2} \int_{0}^{T} \int_{\partial \Omega}\left(n \cdot E_{\varepsilon}\right)^{2} \mathrm{~d} \sigma \mathrm{d} t+\frac{\varepsilon_{0}}{2 \mu} \int_{0}^{T} \int_{\partial \Omega}\left|n \wedge E_{\varepsilon}\right|^{2} \mathrm{~d} \sigma \mathrm{d} t \\
& \varepsilon_{0} \int_{0}^{T} \int_{\partial \Omega} c^{2}\left|\left(n \cdot B_{\varepsilon}\right)\right|\left|n \wedge B_{\varepsilon}\right| \mathrm{d} \sigma \mathrm{d} t \leq \frac{\mu \varepsilon_{0}}{2} \int_{0}^{T} \int_{\partial \Omega} c^{2}\left(n \cdot B_{\varepsilon}\right)^{2} \mathrm{~d} \sigma \mathrm{d} t+\frac{\varepsilon_{0}}{2 \mu} \int_{0}^{T} \int_{\partial \Omega} c^{2}\left|n \wedge B_{\varepsilon}\right|^{2} \mathrm{~d} \sigma \mathrm{d} t
\end{aligned}
$$

with $\mu>0$ small enough. The estimates (38), (39) follow easily since by Propositions

2.1, 2.6 we have

$\int_{0}^{T} \int_{\partial \Omega} \int_{\mathbb{R}^{3}}|(v(p) \cdot n(x))||p| f_{\varepsilon} \mathrm{d} p \mathrm{~d} \sigma \mathrm{d} t \leq C \int_{0}^{T} \int_{\partial \Omega} \int_{\mathbb{R}^{3}}|(v(p) \cdot n(x))|(1+\mathcal{E}(p)) f_{\varepsilon} \mathrm{d} p \mathrm{~d} \sigma \mathrm{d} t \leq C$ and by Proposition 2.2 we know that

$$
\frac{\varepsilon_{0} c}{2} \int_{0}^{T} \int_{\partial \Omega}\left|n \wedge E_{\varepsilon}\right|^{2}+c^{2}\left|n \wedge B_{\varepsilon}\right|^{2} \mathrm{~d} \sigma \mathrm{d} t \leq K^{-}+\frac{\varepsilon_{0} c}{2} T H .
$$




\section{$3 \quad$ Existence results}

We are ready now to prove the existence of $T$ periodic weak solutions for the Vlasov-Maxwell problem (3), (4), (5), (6), (7): it is a straightforward consequence of the uniform estimates for $T$ periodic solutions $\left(f_{\varepsilon}, E_{\varepsilon}, B_{\varepsilon}\right)_{0<\varepsilon \leq 1}$ with $\delta=1$.

Proof. (of Theorem 1.1) The arguments are standard and are left to the reader. We construct our $T$ periodic solution by taking a weak limit point of $\left(f_{\varepsilon}, E_{\varepsilon}, B_{\varepsilon}\right)_{0<\varepsilon \leq 1}$. We only justify that the limit solution satisfies the specular boundary condition (4) and the mass constraints (10). Take $\left(\varepsilon_{k}\right)_{k}$ a sequence of positive numbers converging towards 0 such that

$$
\begin{gathered}
f_{\varepsilon_{k}} \rightarrow f \text { weakly } \star \text { in } L^{\infty}\left(\mathbb{R} \times \Omega \times \mathbb{R}^{3}\right) \\
\left(E_{\varepsilon_{k}}, B_{\varepsilon_{k}}\right) \rightarrow(E, B) \text { weakly in } L_{\text {loc }}^{2}\left(\mathbb{R} ; L^{2}(\Omega)^{6}\right) \\
\left(n \wedge E_{\varepsilon_{k}}, n \wedge B_{\varepsilon_{k}}\right) \rightarrow(n \wedge E, n \wedge B) \text { weakly in } L_{\mathrm{loc}}^{2}\left(\mathbb{R} ; L^{2}(\partial \Omega)^{6}\right) \\
\left(n \cdot E_{\varepsilon_{k}}, n \cdot B_{\varepsilon_{k}}\right) \rightarrow(n \cdot E, n \cdot B) \text { weakly in } L_{\mathrm{loc}}^{2}\left(\mathbb{R} ; L^{2}(\partial \Omega)^{2}\right) .
\end{gathered}
$$

Here $n \wedge$ is the tangential trace and $n$. is the normal trace over $\partial \Omega$. For any $T$ periodic function $\theta \in C^{1}\left(\mathbb{R} \times \bar{\Omega} \times \mathbb{R}^{3}\right)$ satisfying $\theta(t, x, p)=\theta(t, x, R(x) p),(t, x, p) \in \mathbb{R} \times \Sigma^{+}$we have

$\int_{0}^{T} \int_{\Omega} \int_{\mathbb{R}^{3}}\left(\partial_{t} \theta+v \cdot \nabla_{x} \theta+q\left(E_{\varepsilon_{k}}+v \wedge B_{\varepsilon_{k}}\right) \cdot \nabla_{p} \theta\right) f_{\varepsilon_{k}} \mathrm{~d} p \mathrm{~d} x \mathrm{~d} t=\int_{0}^{T} \int_{\partial \Omega} \int_{\mathbb{R}^{3}}(v \cdot n) \theta f_{\varepsilon_{k}} \mathrm{~d} p \mathrm{~d} \sigma \mathrm{d} t .(45)$

But as in the proof of Lemma 2.1 we can write

$$
\begin{aligned}
\int_{0}^{T} \int_{\partial \Omega} \int_{\mathbb{R}^{3}}(v(p) \cdot n(x)) \theta f_{\varepsilon_{k}} \mathrm{~d} p \mathrm{~d} \sigma \mathrm{d} t & =\varepsilon_{k} \int_{0}^{T} \int_{\partial \Omega} \int_{\mathbb{R}^{3}}(v(p) \cdot n(x))_{+} \theta f_{\varepsilon_{k}} \mathrm{~d} p \mathrm{~d} \sigma \mathrm{d} t \\
& -\varepsilon_{k} \int_{0}^{T} \int_{\partial \Omega} \int_{\mathbb{R}^{3}}(v(p) \cdot n(x))_{-} \theta g \mathrm{~d} p \mathrm{~d} \sigma \mathrm{d} t
\end{aligned}
$$

and therefore

$$
\lim _{k \rightarrow+\infty} \int_{0}^{T} \int_{\partial \Omega} \int_{\mathbb{R}^{3}}(v(p) \cdot n(x)) \theta f_{\varepsilon_{k}} \mathrm{~d} p \mathrm{~d} \sigma \mathrm{d} t=0 .
$$

We intend to pass to the limit for $k \rightarrow+\infty$ in (45). As usual we use the compactness average result of DiPerna and Lions [9] (which adapts easily in the time periodic case 
and for bounded domains) in order to treat the non linear terms $f_{\varepsilon_{k}}\left(E_{\varepsilon_{k}}+v \wedge B_{\varepsilon_{k}}\right) \cdot \nabla_{p} \theta$. Finally one gets

$$
\int_{0}^{T} \int_{\Omega} \int_{\mathbb{R}^{3}}\left(\partial_{t} \theta+v(p) \cdot \nabla_{x} \theta+q(E+v(p) \wedge B) \cdot \nabla_{p} \theta\right) f \mathrm{~d} p \mathrm{~d} x \mathrm{~d} t=0
$$

for any $T$ periodic function $\theta \in C^{1}\left(\mathbb{R} \times \bar{\Omega} \times \mathbb{R}^{3}\right)$ satisfying $\theta(t, x, p)=\theta(t, x, R(x) p)$, $(t, x, p) \in \mathbb{R} \times \Sigma^{+}$. We have by Propositions 2.1, 2.6

$$
\int_{0}^{T} \int_{\Sigma^{ \pm}}|(v(p) \cdot n(x))| \gamma^{ \pm} f_{\varepsilon_{k}} \mathrm{~d} p \mathrm{~d} \sigma \mathrm{d} t=M^{-}
$$

and

$$
\sup _{k \in \mathbb{N}} \int_{0}^{T} \int_{\Sigma^{ \pm}}|(v(p) \cdot n(x))| \mathcal{E}(p) \gamma^{ \pm} f_{\varepsilon_{k}} \mathrm{~d} p \mathrm{~d} \sigma \mathrm{d} t<+\infty .
$$

After extraction eventually, we can assume that

$$
\gamma^{ \pm} f_{\varepsilon_{k}} \rightarrow \gamma^{ \pm} f \text { weakly } \star \text { in } L^{\infty}\left(\mathbb{R} \times \Sigma^{ \pm}\right)
$$

and we obtain easily that

$$
\int_{0}^{T} \int_{\Sigma^{ \pm}}|(v(p) \cdot n(x))| \gamma^{ \pm} f \mathrm{~d} p \mathrm{~d} \sigma \mathrm{d} t=M^{-}
$$

Corollary 3.1 Under the hypotheses of Theorem 1.1 (with $\delta=1$ ), the tangential traces of the electro-magnetic field $(E, B)$ satisfies

$$
n \wedge E=n \wedge \nabla_{\tau} h_{2}, \quad c n \wedge(n \wedge B)=\nabla_{\tau} h_{1}
$$

where $h=\nabla_{\tau} h_{1}+n \wedge \nabla_{\tau} h_{2}$,

Proof. With the notations in the proof of Theorem 1.1 we know by Proposition 2.6 that

$$
\sup _{k \in \mathbb{N}} \frac{1}{\varepsilon_{k}} \int_{0}^{T} \int_{\partial \Omega}\left|n \wedge E_{\varepsilon_{k}}-n \wedge \nabla_{\tau} h_{2}\right|^{2}+\left|c n \wedge\left(n \wedge B_{\varepsilon_{k}}\right)-\nabla_{\tau} h_{1}\right|^{2} \mathrm{~d} \sigma \mathrm{d} t<+\infty
$$

which implies that

$$
\lim _{k \rightarrow+\infty}\left(n \wedge E_{\varepsilon_{k}}, c n \wedge\left(n \wedge B_{\varepsilon_{k}}\right)\right)=\left(n \wedge \nabla_{\tau} h_{2}, \nabla_{\tau} h_{1}\right) \text { strongly in } L_{\text {loc }}^{2}\left(\mathbb{R} ; L^{2}(\partial \Omega)^{6}\right)
$$

Therefore we have $(n \wedge E, c n \wedge(n \wedge B))=\left(n \wedge \nabla_{\tau} h_{2}, \nabla_{\tau} h_{1}\right)$. 
We investigate now the Vlasov-Maxwell problem (3), (4), (5), (6) with the perfect conducting boundary condition (11). In order to construct $T$ periodic solutions for this problem we replace (4) by (12) and (11) by (17) with $\delta=\varepsilon \in] 0,1]$ and $h=0$

$$
n \wedge E+\varepsilon c n \wedge(n \wedge B)=0, \quad(t, x) \in \mathbb{R} \times \partial \Omega
$$

Proof. (of Theorem 1.2) For any $\varepsilon \in] 0,1]$ we denote by $\left(f_{\varepsilon}, E_{\varepsilon}, B_{\varepsilon}\right)$ a $T$ periodic weak solution for (3), (12), (5), (6), (46). By Proposition 2.1 we know that

$$
\left.\left.\int_{0}^{T} \int_{\partial \Omega} \int_{\mathbb{R}^{3}}(v(p) \cdot n(x))_{ \pm} f_{\varepsilon}(t, x, p) \mathrm{d} p \mathrm{~d} \sigma \mathrm{d} t=M^{-}, \quad \varepsilon \in\right] 0,1\right] .
$$

Notice also that by Proposition 2.2 we have

$\varepsilon \int_{0}^{T} \int_{\partial \Omega} \int_{\mathbb{R}^{3}}(v(p) \cdot n(x))_{+} \mathcal{E}(p) f_{\varepsilon} \mathrm{d} p \mathrm{~d} \sigma \mathrm{d} t+\frac{\varepsilon_{0} c}{2 \varepsilon} \int_{0}^{T} \int_{\partial \Omega}\left|n \wedge E_{\varepsilon}\right|^{2}+\varepsilon^{2} c^{2}\left|n \wedge B_{\varepsilon}\right|^{2} \mathrm{~d} \sigma \mathrm{d} t=\varepsilon K^{-}$ implying that

$$
\int_{0}^{T} \int_{\partial \Omega} \int_{\mathbb{R}^{3}}(v(p) \cdot n(x))_{+} \mathcal{E}(p) f_{\varepsilon} \mathrm{d} p \mathrm{~d} \sigma \mathrm{d} t \leq K^{-}
$$

and

$$
\frac{\varepsilon_{0} c}{2} \int_{0}^{T} \int_{\partial \Omega}\left|n \wedge E_{\varepsilon}\right|^{2}+\varepsilon^{2} c^{2}\left|n \wedge B_{\varepsilon}\right|^{2} \mathrm{~d} \sigma \mathrm{d} t \leq \varepsilon^{2} K^{-} .
$$

From the above inequality we deduce that $\left(n \wedge E_{\varepsilon}\right)_{\varepsilon}$ converges towards 0 in $L_{\text {loc }}^{2}\left(\mathbb{R} ; L^{2}(\partial \Omega)^{3}\right)$. Combining (12) and (48) yields for any $\varepsilon \in] 0,1]$

$$
\begin{aligned}
\int_{0}^{T} \int_{\partial \Omega} \int_{\mathbb{R}^{3}}(v \cdot n)_{-} \mathcal{E} f_{\varepsilon} \mathrm{d} p \mathrm{~d} \sigma \mathrm{d} t & =\int_{0}^{T} \int_{\partial \Omega} \int_{\mathbb{R}^{3}}(v \cdot n)_{-} \mathcal{E}\left(\varepsilon g+(1-\varepsilon) f_{\varepsilon}(t, x, R(x) p)\right) \mathrm{d} p \mathrm{~d} \sigma \mathrm{d} t \\
& \leq \varepsilon \int_{0}^{T} \int_{\partial \Omega} \int_{\mathbb{R}^{3}}(v \cdot n)_{-} \mathcal{E}(p) g \mathrm{~d} p \mathrm{~d} \sigma \mathrm{d} t+(1-\varepsilon) K^{-} \\
& =K^{-}
\end{aligned}
$$

At this stage let us mention that (38), (39) still hold true uniformly with respect to $\varepsilon \in] 0,1]$. Indeed, this is a direct consequence of (37) (which is valid for any $\delta=\varepsilon \in] 0,1]$ ) since we already know that

$$
\begin{aligned}
\int_{0}^{T} \int_{\partial \Omega} \int_{\mathbb{R}^{3}}((v(p) \cdot n(x)))_{ \pm}|p| f_{\varepsilon} \mathrm{d} p \mathrm{~d} \sigma \mathrm{d} t & \leq C \int_{0}^{T} \int_{\partial \Omega} \int_{\mathbb{R}^{3}}(v(p) \cdot n(x))_{ \pm}(1+\mathcal{E}(p)) f_{\varepsilon} \mathrm{d} p \mathrm{~d} \sigma \mathrm{d} t \\
& \leq C\left(M^{-}+K^{-}\right)
\end{aligned}
$$

and

$$
\frac{\varepsilon_{0} c}{2} \int_{0}^{T} \int_{\partial \Omega}\left|n \wedge E_{\varepsilon}\right|^{2}+c^{2}\left|n \wedge B_{\varepsilon}\right|^{2} \mathrm{~d} \sigma \mathrm{d} t \leq 2 K^{-} .
$$


Therefore we obtain uniform bounds for the total energy and the normal traces of the electro-magnetic field. From now on the arguments are similar to those in the proof of Theorem 1.1. Taking $\left(\varepsilon_{k}\right)_{k}$ a sequence of positive numbers converging towards 0 such that

$$
\begin{gathered}
f_{\varepsilon_{k}} \rightarrow f \text { weakly } \star \text { in } L^{\infty}\left(\mathbb{R} \times \Omega \times \mathbb{R}^{3}\right) \\
\left(E_{\varepsilon_{k}}, B_{\varepsilon_{k}}\right) \rightarrow(E, B) \text { weakly in } L_{\text {loc }}^{2}\left(\mathbb{R} ; L^{2}(\Omega)^{6}\right) \\
\left(n \wedge E_{\varepsilon_{k}}, n \wedge B_{\varepsilon_{k}}\right) \rightarrow(n \wedge E, n \wedge B) \text { weakly in } L_{\mathrm{loc}}^{2}\left(\mathbb{R} ; L^{2}(\partial \Omega)^{6}\right) \\
\left(n \cdot E_{\varepsilon_{k}}, n \cdot B_{\varepsilon_{k}}\right) \rightarrow(n \cdot E, n \cdot B) \text { weakly in } L_{\mathrm{loc}}^{2}\left(\mathbb{R} ; L^{2}(\partial \Omega)^{2}\right) .
\end{gathered}
$$

it is easily seen that $(f, E, B)$ is a $T$ periodic weak solution of $(3),(4),(5),(6)$. Notice also that by (49) we have $\lim _{k \rightarrow+\infty} n \wedge E_{\varepsilon_{k}}=0$ strongly in $L_{\text {loc }}^{2}\left(\mathbb{R} ; L^{2}(\partial \Omega)^{3}\right)$, saying that the electric field $E$ satisfies the perfect conducting boundary condition $n \wedge E=0$ on $\mathbb{R} \times \partial \Omega$.

\section{References}

[1] A. Arseneev, Global existence of a weak solution of the Vlasov system of equations, U.R.S.S. Comp. and Math. Phys. 15 (1975) 131-143.

[2] C. Bardos and P. Degond, Global existence for the Vlasov-Poisson equation in three space variables with small initial data, Ann. Inst. H. Poincaré, Anal. non linéaire 2 (1985) 101-118.

[3] N. Ben Abdallah, Weak solutions of the initial-boundary value problem for the Vlasov-Poisson system, Math. Meth. Appl. Sci. 17 (1994) 451-476.

[4] M. Bostan, Solutions périodiques en temps des équations de Vlasov-Maxwell, C. R. Acad. Sci. Paris, Sér. I 339 (2004) 451-456.

[5] M. Bostan, Asymptotic behavior of weak solutions for the relativistic VlasovMaxwell equations with large light speed, J. Diff. Eq. 227 (2006) 444-498.

[6] M. Bostan, Boundary value problem for the three dimensional time periodic Vlasov-Maxwell system, J. Comm. Math. Sci. 3 (2005) 621-663 . 
[7] F. Bouchut, F. Golse and C. Pallard, Classical solutions and the Glassey-Strauss theorem for the 3D Vlasov-Maxwell system, Arch. Rational Mech. Anal. 170 (2003) $1-15$.

[8] P. Degond, Local existence of solutions of the Vlasov-Maxwell equations and convergence to the Vlasov-Poisson equations for infinite light velocity, Math. Meth. Appl. Sci. 8 (1986) 533-558.

[9] R. J. Diperna and P. L. Lions, Global weak solutions of the Vlasov-Maxwell system, Comm. Pure Appl. Math. XVII (1989) 729-757.

[10] V. Girault, P.-A. Raviart, Finite element approximation of the Navier-Stokes equations, Lecture Notes in Math., Springer-Verlag, 1979.

[11] R. Glassey and J. Schaeffer, The relativistic Vlasov-Maxwell system in two space dimensions I, Arch. Rational Mech. Anal. 141 (1998) 331-354.

[12] R. Glassey and J. Schaeffer, The relativistic Vlasov-Maxwell system in two space dimensions II, Arch. Rational Mech. Anal. 141 (1998) 355-374.

[13] R. Glassey and W. Strauss, Singularity formation in a collisionless plasma could only occur at high velocities, Arch. Rational Mech. Anal. 92 (1986) 56-90.

[14] R. Glassey and W. Strauss, Large velocities in the relativistic Vlasov-Maxwell equations, J. Fac. Sci. Tokyo 36 (1989) 615-627.

[15] C. Greengard and P.-A. Raviart, A boundary value problem for the stationary Vlasov-Poisson equations : the plane diode, Comm. Pure and Appl. Math. vol. XLIII (1990) 473-507.

[16] Y. Guo, Global weak solutions of the Vlasov-Maxwell system with boundary conditions, Comm. Math. Phys. 154 (1993) 245-263.

[17] E. Horst and R. Hunze, Weak solutions of the initial value problem for the unmodified nonlinear Vlasov equation, Math. Meth. in the Appl. Sci. 6 (1984) 262-279.

[18] S. Klainerman and G. Staffilani, A new approach to study the Vlasov-Maxwell system, Commun. Pure Appl. Anal. 1 (2002) 103-125. 
[19] H. Lee, The classical limit of the relativistic Vlasov-Maxwell system in two space dimensions, Math. Methods Appl. Sci. 27(2004) 249-287.

[20] P.-L. Lions and B. Perthame, Propagation of moments and regularity for the 3dimensional Vlasov-Poisson system, Invent. Math. 105 (1991) 415-430.

[21] J. Necas, Les méthodes directes en théorie des équations elliptiques, Masson, 1967.

[22] K. Pfaffelmoser, Global classical solutions of the Vlasov-Poisson system in three dimensions for general initial data, J. Diff. Eq. 95 (1992) 281-303.

[23] F. Poupaud, Boundary value problems for the stationary Vlasov-Maxwell system, Forum Math. 4 (1992) 499-527.

[24] J. Schaeffer, The classical limit of the relativistic Vlasov-Maxwell system, Comm. Math. Phys. 104 (1986) 403-421.

[25] J. Schaeffer, Global existence of smooth solutions to the Vlasov-Poisson system in three dimensions, Comm. P.D.E. 16 (1991) 1313-1335.

[26] T. Ukai and S. Okabe, On the classical solution in the large time of the two dimensional Vlasov equations, Osaka J. Math. 15 (1978) 245-261. 